\title{
Finding Modular Functions for Ramanujan-Type Identities
}

\author{
William Y.C. Chen ${ }^{1}$, Julia Q.D. Du ${ }^{2}$ and Jack C.D. Zhao ${ }^{3}$ \\ ${ }^{1,2}$ Center for Applied Mathematics \\ Tianjin University \\ Tianjin 300072, P. R. China \\ ${ }^{3}$ Center for Combinatorics \\ Nankai University \\ Tianjin 300071, P. R. China \\ Emails: ${ }^{1}$ chenyc@tju.edu.cn, ${ }^{2}$ qddu@tju.edu.cn, ${ }^{3}$ cdzhao@mail.nankai.edu.cn \\ Dedicated to Professor George E. Andrews on the occasion of his 80th birthday
}

\begin{abstract}
This paper is concerned with a class of partition functions $a(n)$ introduced by Radu and defined in terms of eta-quotients. By utilizing the transformation laws of Newman, Schoeneberg and Robins, and Radu's algorithms, we present an algorithm to find Ramanujan-type identities for $a(m n+t)$. While this algorithm is not guaranteed to succeed, it applies to many cases. For example, we deduce a witness identity for $p(11 n+6)$ with integer coefficients. Our algorithm also leads to Ramanujan-type identities for the overpartition functions $\bar{p}(5 n+2)$ and $\bar{p}(5 n+3)$ and AndrewsPaule's broken 2-diamond partition functions $\triangle_{2}(25 n+14)$ and $\triangle_{2}(25 n+24)$. It can also be extended to derive Ramanujan-type identities on a more general class of partition functions. For example, it yields the Ramanujan-type identities on Andrews' singular overpartition functions $\bar{Q}_{3,1}(9 n+3)$ and $\bar{Q}_{3,1}(9 n+6)$ due to Shen, the 2-dissection formulas of Ramanujan and the 8-dissection formulas due to Hirschhorn.
\end{abstract}

AMS Classification. 05A15, 11P83, 11P84, 05A17.

Keywords. Ramanujan-type identities, Modular functions, Generalized eta-functions, Partition functions. 


\section{Introduction}

Throughout this paper, we follow the standard $q$-series notation in [16]:

$$
(a ; q)_{\infty}=\prod_{n=0}^{\infty}\left(1-a q^{n}\right) \quad \text { and } \quad\left(a_{1}, a_{2}, \ldots, a_{m} ; q\right)_{\infty}=\prod_{j=1}^{m}\left(a_{j} ; q\right)_{\infty}
$$

where $|q|<1$. In the study of congruence properties and identities on partition functions, Radu [35-37] defined a class of partition functions $a(n)$ by

$$
\sum_{n=0}^{\infty} a(n) q^{n}=\prod_{\delta \mid M}\left(q^{\delta} ; q^{\delta}\right)_{\infty}^{r_{\delta}},
$$

where $M$ is a positive integer and $r_{\delta}$ are integers. Many partition functions fall into the framework of the above definition of $a(n)$, such as the partition function $p(n)$, the overpartition function $\bar{p}(n)$ [11], the Ramanujan $\tau$-function [18,19,39], the $k$-colored partition functions, the $t$-core partition functions, the 2-colored Frobenius partition functions and the broken $k$-diamond partition functions $\Delta_{k}(n)$ [4].

In this paper, we aim to present an algorithm to compute the generating function

$$
\sum_{n=0}^{\infty} a(m n+t) q^{n}
$$

for fixed $m>0$ and $0 \leq t \leq m-1$ by finding suitable modular functions for $\Gamma_{1}(N)$. When $M=1$ and $r_{1}=-1, a(n)$ specializes to the partition function $p(n)$. Kolberg [26] proved that for a positive integer $m$ prime to 6 , and $0 \leq t \leq m-1$,

$$
\sum_{n=0}^{\infty} p(m n+t) q^{m n+t}=(-1)^{(m-1) t} \frac{\left(q^{m^{2}} ; q^{m^{2}}\right)_{\infty}}{\left(q^{m} ; q^{m}\right)_{\infty}^{m+1}} \operatorname{det} M_{t},
$$

where $M_{t}=\left(g_{-t-i+j}\right)_{(m-1) \times(m-1)}$,

$$
g_{t}=\sum_{\frac{1}{2} n(3 n+1) \equiv t(\bmod m)}(-1)^{n} q^{\frac{1}{2} n(3 n+1)},
$$

and $g_{t}=g_{s}$ when $t \equiv s(\bmod m)$. In view of (1.3), he derived some identities on $p(n)$, for example,

$$
\sum_{n=0}^{\infty} p(5 n) q^{n}=\frac{\left(q^{5} ; q^{5}\right)_{\infty}}{(q ; q)_{\infty}^{2}\left(q, q^{4} ; q^{5}\right)_{\infty}^{8}}-3 q \frac{\left(q^{5} ; q^{5}\right)_{\infty}^{6}\left(q, q^{4} ; q^{5}\right)_{\infty}^{2}}{(q ; q)_{\infty}^{7}}
$$

and

$$
\left(\sum_{n=0}^{\infty} p(5 n) q^{n}\right)\left(\sum_{n=0}^{\infty} p(5 n+3) q^{n}\right)=3 \frac{\left(q^{5} ; q^{5}\right)_{\infty}^{4}}{(q ; q)_{\infty}^{6}}+25 q \frac{\left(q^{5} ; q^{5}\right)_{\infty}^{10}}{(q ; q)_{\infty}^{12}}
$$

Atkin and Swinnerton-Dyer [5] have shown that $g_{t}$ can always be expressed by certain infinite products for $m>3$. Then the left hand side of (1.3) can be expressed in terms of certain infinite products. Kolberg pointed out that when $m>5$, this becomes much more complicated. For $m=11,13$, Bilgici and Ekin $[7,8]$ used the method of Kolberg to compute the generating function

$$
\sum_{n=0}^{\infty} p(m n+t) q^{m n+t}
$$


for all $0 \leq t \leq m-1$.

Based on the ideas of Rademacher [33], Newman [28,29] and Kolberg [26], Radu [37] developed an algorithm to verify the congruences

$$
a(m n+t) \equiv 0 \quad(\bmod u),
$$

for any given $m, t$ and $u$, and for all $n \geq 0$, where $a(n)$ is defined in (1.1). Moreover, Radu [35] developed an algorithm, called the Ramanujan-Kolberg algorithm, to derive identities on the generating functions of $a(m n+t)$ using modular functions for $\Gamma_{0}(N)$. A description of the Ramanujan-Kolberg algorithm can be found in Paule and Radu [32]. Smoot [46] developed a Mathematica package RaduRK to implement Radu's algorithm. It should be mentioned that Eichhorn [13] extended the technique in $[14,15]$ to partition functions $a(n)$ defined by

$$
\sum_{n=0}^{\infty} a(n) q^{n}=\prod_{j=1}^{L}\left(q^{j} ; q^{j}\right)_{\infty}^{e_{j}},
$$

where $L$ is a positive integer and $e_{j}$ are integers, and reduced the verification of the congruences (1.5) to a finite number of cases. It is easy to see that the defining relations (1.1) and (1.6) are equivalent to each other. In this paper, we shall adopt the form of (1.1) in accordance with the notation of eta-quotients.

Recall that the Dedekind eta-function $\eta(\tau)$ is defined by

$$
\eta(\tau)=q^{\frac{1}{24}} \prod_{n=1}^{\infty}\left(1-q^{n}\right),
$$

where $q=e^{2 \pi i \tau}, \tau \in \mathbb{H}=\{\tau \in \mathbb{C}: \operatorname{Im}(\tau)>0\}$. An eta-quotient is a function of the form

$$
\prod_{\delta \mid M} \eta^{r_{\delta}}(\delta \tau)
$$

where $M \geq 1$ and each $r_{\delta}$ is an integer.

The Ramanujan-Kolberg algorithm leads to verifications of some identities on $p(n)$ due to Ramanujan [38], Zuckerman [50] and Kolberg [26], for example,

$$
\sum_{n=0}^{\infty} p(5 n+4) q^{n}=5 \frac{\left(q^{5} ; q^{5}\right)_{\infty}^{5}}{(q ; q)_{\infty}^{6}},
$$

see [38, eq. (18)]. It should be noted that there are some Ramanujan-type identities that are not covered by the Ramanujan-Kolberg algorithm, such as the identity (1.4).

In this paper, we develop an algorithm to derive Ramanujan-type identities for $a(m n+t)$ for $m>0$ and $0 \leq t \leq m-1$, which is essentially a modified version of Radu's algorithm. We first find a necessary and sufficient condition for a product of a generalized eta-quotient and the generating function (1.2) to be a modular function for $\Gamma_{1}(N)$ up to a power of $q$. Then we try to express this modular function as a linear combination of generalized eta-quotients over $\mathbb{Q}$.

For example, our algorithm leads to a verification of (1.4) for $p(5 n)$. Moreover, we obtain Ramanujan-type identities for the overpartition functions $\bar{p}(5 n+2)$ and $\bar{p}(5 n+3)$ and the broken 2-diamond partition functions $\Delta_{2}(25 n+14)$ and $\Delta_{2}(25 n+24)$. We also obtain the following witness identity with integer coefficients for $p(11 n+6)$. 
Theorem 1.1. We have

$$
\begin{aligned}
& z_{0} \sum_{n=0}^{\infty} p(11 n+6) q^{n} \\
& =11 z^{10}+121 z^{8} e+330 z^{9}-484 z^{7} e-990 z^{8}+484 z^{6} e+792 z^{7} \\
& \quad-484 z^{5} e+44 z^{6}+1089 z^{4} e-132 z^{5}-1452 z^{3} e-451 z^{4} \\
& \quad+968 z^{2} e+748 z^{3}-242 z e-429 z^{2}+77 z+11
\end{aligned}
$$

where

$$
\begin{aligned}
& z_{0}=\frac{(q ; q)_{\infty}^{24}}{q^{20}\left(q^{11} ; q^{11}\right)_{\infty}^{23}\left(q, q^{10} ; q^{11}\right)_{\infty}^{28}\left(q^{2}, q^{9} ; q^{11}\right)_{\infty}^{16}\left(q^{3}, q^{8} ; q^{11}\right)_{\infty}^{12}\left(q^{4}, q^{7} ; q^{11}\right)_{\infty}^{4}} \\
& z=\frac{(q ; q)_{\infty}}{q^{2}\left(q^{11} ; q^{11}\right)_{\infty}\left(q, q^{10} ; q^{11}\right)_{\infty}^{3}\left(q^{2}, q^{9} ; q^{11}\right)_{\infty}^{2}} \\
& e=\frac{(q ; q)_{\infty}^{3}}{q^{3}\left(q^{11} ; q^{11}\right)_{\infty}^{3}\left(q, q^{10} ; q^{11}\right)_{\infty}^{5}\left(q^{2}, q^{9} ; q^{11}\right)_{\infty}^{5}\left(q^{3}, q^{8} ; q^{11}\right)_{\infty}^{4}\left(q^{4}, q^{7} ; q^{11}\right)_{\infty}}
\end{aligned}
$$

Bilgici and Ekin [8] deduced a witness identity for $p(11 n+6)$ with integer coefficients using the method of Kolberg. Radu [35] obtained a witness identity for $p(11 n+6)$ by using the RamanujanKolberg algorithm. Hemmecke [20] generalized Radu's algorithm and derived a witness identity for $p(11 n+6)$. Paule and Radu [31] found a polynomial relation on the generating function of $p(11 n+6)$, which can also be viewed as a witness identity. Moreover, Paule and Radu [30] found a witness identity for $p(11 n+6)$ in terms of eta-quotients and the $U_{2}$-operator acting on eta-quotients.

Our algorithm can be extended to a more general class of partition functions $b(n)$ defined by

$$
\sum_{n=0}^{\infty} b(n) q^{n}=\prod_{\delta \mid M}\left(q^{\delta} ; q^{\delta}\right)_{\infty}^{r_{\delta}} \prod_{\substack{\delta \mid M \\ 0<g<\delta}}\left(q^{g}, q^{\delta-g} ; q^{\delta}\right)_{\infty}^{r_{\delta, g}},
$$

where $M$ is a positive integer and $r_{\delta}, r_{\delta, g}$ are integers. Notice that (1.10) is a generalized eta-quotient up to a power of $q$.

Recall that for a positive integer $\delta$ and a residue class $g(\bmod \delta)$, the generalized Dedekind etafunction $\eta_{\delta, g}(\tau)$ is defined by

$$
\eta_{\delta, g}(\tau)=q^{\frac{\delta}{2} P_{2}\left(\frac{g}{\delta}\right)} \prod_{\substack{n>0 \\ n \equiv g(\bmod \delta)}}\left(1-q^{n}\right) \prod_{\substack{n>0 \\ n \equiv-g(\bmod \delta)}}\left(1-q^{n}\right),
$$

where

$$
P_{2}(t)=\{t\}^{2}-\{t\}+\frac{1}{6}
$$

is the second Bernoulli function and $\{t\}$ is the fractional part of $t$, see, for example, $[41,43]$. Note that

$$
\eta_{\delta, 0}(\tau)=\eta^{2}(\delta \tau) \quad \text { and } \quad \eta_{\delta, \frac{\delta}{2}}(\tau)=\frac{\eta^{2}\left(\frac{\delta}{2} \tau\right)}{\eta^{2}(\delta \tau)}
$$


A generalized eta-quotient is a function of the form

$$
\prod_{\substack{\delta \mid M \\ 0 \leq g<\delta}} \eta_{\delta, g}^{r_{\delta, g}}(\tau)
$$

where $M \geq 1$ and

$$
r_{\delta, g} \in \begin{cases}\frac{1}{2} \mathbb{Z}, & \text { if } g=0 \text { or } g=\frac{\delta}{2}, \\ \mathbb{Z}, & \text { otherwise }\end{cases}
$$

see, for example, Robins [41]. In view of (1.12), when $g=0$ or $g=\frac{\delta}{2}$, if $r_{\delta, g} \in \frac{1}{2} \mathbb{Z}$, then the powers of the eta-functions in (1.13) are integers.

For partition functions $b(n)$ as defined in (1.10), our algorithm can be extended to derive Ramanujantype identities on $b(m n+t)$ for $m>0$ and $0 \leq t \leq m-1$, such as the Ramanujan-type identities on Andrews' singular overpartition functions $\bar{Q}_{3,1}(9 n+3)$ and $\bar{Q}_{3,1}(9 n+6)$ due to Shen [45]. The extended algorithm can also be employed to derive dissection formulas, such as the 2-dissection formulas of Ramanujan, first proved by Andrews [2], and the 8-dissection formulas due to Hirschhorn [22].

\section{Finding Modular Functions for $\Gamma_{1}(N)$}

For the partition functions $a(n)$ as defined by (1.1), namely,

$$
\sum_{n=0}^{\infty} a(n) q^{n}=\prod_{\delta \mid M}\left(q^{\delta} ; q^{\delta}\right)_{\infty}^{r_{\delta}},
$$

where $M$ is a positive integer and $r_{\delta}$ are integers, Radu [37] defined

$$
g_{m, t}(\tau)=q^{\frac{t-\ell}{m}} \sum_{n=0}^{\infty} a(m n+t) q^{n}
$$

where

$$
\ell=-\frac{1}{24} \sum_{\delta \mid M} \delta r_{\delta}
$$

Let $\phi(\tau)$ be a generalized eta-quotient, and let $F(\tau)=\phi(\tau) g_{m, t}(\tau)$. The objective of this section is to give a criterion for $F(\tau)$ to be a modular function for $\Gamma_{1}(N)$. We find that the transformation formula for $g_{m, t}(\tau)$ under $\Gamma_{1}(N)^{*}$ is analogous to the transformation formula of Radu [37, Lemma 2.14] with respect to $\Gamma_{0}(N)^{*}$. Then we utilize the transformation laws of Newman [29] and Robins [41] to obtain the transformation formula of $F(\tau)$. With the aid of the Laurent expansions of $\phi(\tau)$ and $g_{m, t}(\tau)$, we obtain a necessary and sufficient condition for $F(\tau)$ to be a modular function for $\Gamma_{1}(N)$.

We first state the conditions on $N$. In fact, we make the following changes on the conditions on $N$ given by Definition 34 and Definition 35 in [35]: Change the condition $\delta \mid m N$ for every $\delta \mid M$ with $r_{\delta} \neq 0$ to $M \mid N$, and add the following condition 7. For completeness, we list all the conditions on $N$. Let $\kappa=\operatorname{gcd}\left(m^{2}-1,24\right)$. Assume that $N$ satisfies the following conditions:

1. $M \mid N$. 
2. $p \mid N$ for any prime $p \mid m$.

3. $\kappa N \sum_{\delta \mid M} r_{\delta} \equiv 0(\bmod 8)$.

4. $\kappa m N^{2} \sum_{\delta \mid M} \frac{r_{\delta}}{\delta} \equiv 0(\bmod 24)$.

5. $\frac{24 m}{\operatorname{gcd}(\kappa \alpha(t), 24 m)} \mid N$, where $\alpha(t)=-\sum_{\delta \mid M} \delta r_{\delta}-24 t$.

6. Let $\prod_{\delta \mid M} \delta^{\left|r_{\delta}\right|}=2^{z} j$, where $z \in \mathbb{N}$ and $j$ is odd. If $2 \mid m$, then $\kappa N \equiv 0(\bmod 4)$ and $N z \equiv 0$ $(\bmod 8)$, or $z \equiv 0(\bmod 2)$ and $N(j-1) \equiv 0(\bmod 8)$.

7. Let $\mathbb{S}_{n}=\left\{j^{2}(\bmod n): j \in \mathbb{Z}_{n}, \operatorname{gcd}(j, n)=1, j \equiv 1(\bmod N)\right\}$. For any $s \in \mathbb{S}_{24 m M}$,

$$
\frac{s-1}{24} \sum_{\delta \mid M} \delta r_{\delta}+t s \equiv t \quad(\bmod m) .
$$

Note that there always exists $N$ satisfying the above conditions, because $N=24 m M$ would make a feasible choice. From now on, we denote by $\gamma$ the matrix $\left(\begin{array}{ll}a & b \\ c & d\end{array}\right)$.

Theorem 2.1. For a given partition function $a(n)$ as defined by (1.1), and for given integers $m$ and $t$, suppose that $N$ is a positive integer satisfying the conditions 1-7. Let

$$
F(\tau)=\phi(\tau) g_{m, t}(\tau)
$$

where

$$
\phi(\tau)=\prod_{\delta \mid N} \eta^{a_{\delta}}(\delta \tau) \prod_{\substack{\delta \mid N \\ 0<g \leq\lfloor\delta / 2\rfloor}} \eta_{\delta, g}^{a_{\delta, g}}(\tau),
$$

and $a_{\delta}$ and $a_{\delta, g}$ are integers. Then $F(\tau)$ is a modular function with respect to $\Gamma_{1}(N)$ if and only if $a_{\delta}$ and $a_{\delta, g}$ satisfy the following conditions:

(1) $\sum_{\delta \mid N} a_{\delta}+\sum_{\delta \mid M} r_{\delta}=0$,

(2) $N \sum_{\delta \mid N} \frac{a_{\delta}}{\delta}+2 N \sum_{\substack{\delta \mid N \\ 0<g \leq\lfloor\delta / 2\rfloor}} \frac{a_{\delta, g}}{\delta}+N m \sum_{\delta \mid M} \frac{r_{\delta}}{\delta} \equiv 0(\bmod 24)$,

(3) $\sum_{\delta \mid N} \delta a_{\delta}+12 \sum_{\substack{\delta \leq N \\ 0<g \leq\lfloor\delta / 2\rfloor}} \delta P_{2}\left(\frac{g}{\delta}\right) a_{\delta, g}+m \sum_{\delta \mid M} \delta r_{\delta}+\frac{\left(m^{2}-1\right) \alpha(t)}{m} \equiv 0(\bmod 24)$,

(4) For any integer $0<a<12 N$ with $\operatorname{gcd}(a, 6)=1$ and $a \equiv 1(\bmod N)$,

$$
\prod_{\delta \mid N}\left(\frac{\delta}{a}\right)^{\left|a_{\delta}\right|} \prod_{\delta \mid M}\left(\frac{m \delta}{a}\right)^{\left|r_{\delta}\right|} e^{\sum_{\delta \mid N} \sum_{g=1}^{\lfloor\delta / 2\rfloor} \pi i\left(\frac{g}{\delta}-\frac{1}{2}\right)(a-1) a_{\delta, g}}=1 .
$$


For example, consider the overpartition function $\bar{p}(n)$. Recall that an overpartition of a positive integer $n$ is a partition of $n$ where the first occurrence of each distinct part may be overlined, and the number of overpartitions of $n$ is denoted by $\bar{p}(n)$ for $n \geq 1$ and $\bar{p}(0)=1$. As noted by Corteel and Lovejoy [11], the generating function of $\bar{p}(n)$ is given by

$$
\sum_{n=0}^{\infty} \bar{p}(n) q^{n}=\frac{\left(q^{2} ; q^{2}\right)_{\infty}}{(q ; q)_{\infty}^{2}}
$$

For the overpartition function $\bar{p}(5 n+2)$, we see that $N=10$ satisfies the conditions $1-7$. Next we proceed to find a generalized eta-quotient $\phi(\tau)$ such that $\phi(\tau) g_{5,2}(\tau)$ is a modular function for $\Gamma_{1}(10)$. By the above theorem, the function

$$
\prod_{\delta \mid 10} \eta^{a_{\delta}}(\delta \tau) \prod_{\substack{\delta \mid 10 \\ 0<g \leq\lfloor\delta / 2\rfloor}} \eta_{\delta, g}^{a_{\delta, g}}(\tau) g_{5,2}(\tau)
$$

is a modular function for $\Gamma_{1}(10)$ if and only if $a_{\delta}$ and $a_{\delta, g}$ fulfill the following conditions:

$$
\left\{\begin{array}{c}
a_{1}+a_{2}+a_{5}+a_{10}-1=0, \\
10 a_{1}+5 a_{2}+10 a_{2,1}+2 a_{5}+4 a_{5,1}+4 a_{5,2}+a_{10}+2 a_{10,1} \\
+2 a_{10,2}+2 a_{10,3}+2 a_{10,4}+2 a_{10,5}-3 \equiv 0 \quad(\bmod 24), \\
a_{1}+2 a_{2}-2 a_{2,1}+5 a_{5}+\frac{2 a_{5,1}}{5}-\frac{22 a_{5,2}}{5}+10 a_{10}+\frac{46 a_{10,1}}{5} \\
+\frac{4 a_{10,2}}{5}-\frac{26 a_{10,3}}{5}-\frac{44 a_{10,4}}{5}-10 a_{10,5}+\frac{48}{5} \equiv 0 \quad(\bmod 24), \\
\left(\frac{10}{a}\right) \prod_{\delta \mid 10}\left(\frac{\delta}{a}\right)^{\left|a_{\delta}\right|} e^{\sum \sum_{\delta \mid 10}^{\lfloor\delta / 2\rfloor} \pi i\left(\frac{g}{\delta}-\frac{1}{2}\right)(a-1) a_{\delta, g}}=1,
\end{array}\right.
$$

for any $0<a<120$ with $\operatorname{gcd}(a, 6)=1$ and $a \equiv 1(\bmod 10)$. We find that

$$
\begin{aligned}
\left(a_{1}, a_{2}, a_{2,1}, a_{5}, a_{5,1}, a_{5,2}, a_{10}, a_{10,1}, a_{10,2}, a_{10,3}, a_{10,4}, a_{10,5}\right) & \\
= & (0,0,0,0,0,0,1,0,0,0,-8,9)
\end{aligned}
$$

is an integer solution of (2.3). Let

$$
\phi(\tau)=\frac{\eta(10 \tau) \eta_{10,5}^{9}(\tau)}{\eta_{10,4}^{8}(\tau)}
$$

Since

$$
g_{5,2}(\tau)=q^{\frac{2}{5}} \sum_{n=0}^{\infty} \bar{p}(5 n+2) q^{n}
$$

we find that

$$
F(\tau)=q^{\frac{2}{5}} \phi(\tau) \sum_{n=0}^{\infty} \bar{p}(5 n+2) q^{n}
$$

is a modular function with respect to $\Gamma_{1}(10)$. 
Let

$$
\Gamma_{1}(N)^{*}=\left\{\left(\begin{array}{ll}
a & b \\
c & d
\end{array}\right) \in \Gamma_{1}(N): \operatorname{gcd}(a, 6)=1, a c>0\right\} .
$$

The following lemma asserts that the invariance of the function $f(\tau)$ under $\Gamma_{1}(N)$ is equivalent to the invariance under $\Gamma_{1}(N)^{*}$.

Lemma 2.2. Let $k$ be an integer, $N$ be a positive integer and $f: \mathbb{H} \rightarrow \mathbb{C}$ be a function such that

$$
f(\gamma \tau)=(c \tau+d)^{k} f(\tau)
$$

for any $\gamma \in \Gamma_{1}(N)^{*}$. Then $f$ is weight- $k$ invariant under $\Gamma_{1}(N)$.

Proof. Let

$$
A=\left\{\left(\begin{array}{ll}
a & b \\
c & d
\end{array}\right) \in \Gamma_{1}(N): \operatorname{gcd}(a, 6)=1\right\} .
$$

By Lemma 3 of Newman [29], we know that $\Gamma_{1}(N)$ is generated by $A$. Hence it suffices to show that

$$
f(\gamma \tau)=(c \tau+d)^{k} f(\tau)
$$

for any $\gamma \in A$. By the condition of Lemma 2.2, we may restrict our attention only to two cases. (1) $\gamma \in A, a>0$ and $c \leq 0$. (2) $\gamma \in A, a<0$ and $c \geq 0$. Here we only consider the first case, and the second case can be justified in the same manner. For the first case, since $a>0$ and $c \leq 0$, there exists a positive integer $x$ such that $a x+\frac{c}{N}>0$. Let

$$
\gamma_{1}=\left(\begin{array}{cc}
1 & 0 \\
N x & 1
\end{array}\right) \quad \text { and } \quad \gamma_{2}=\left(\begin{array}{cc}
a & b \\
N a x+c & N b x+d
\end{array}\right) .
$$

Then $\gamma_{2}=\gamma_{1} \gamma$ and $\gamma_{1} \in \Gamma_{1}(N)^{*}$. Therefore,

$$
f\left(\gamma_{2} \tau\right)=f\left(\gamma_{1}(\gamma \tau)\right)=(N x(\gamma \tau)+1)^{k} f(\gamma \tau) .
$$

Since $\gamma \in A$, we have $\operatorname{gcd}(a, 6)=1$, and so $\gamma_{2} \in \Gamma_{1}(N)^{*}$. Applying (2.5) with $\gamma_{2} \in \Gamma_{1}(N)^{*}$, we get

$$
f\left(\gamma_{2} \tau\right)=((N a x+c) \tau+(N b x+d))^{k} f(\tau) .
$$

Combining (2.6) and (2.7), we deduce that

$$
f(\gamma \tau)=(c \tau+d)^{k} f(\tau),
$$

as claimed.

The following transformation formula for $g_{m, t}(\tau)$ under $\Gamma_{1}(N)^{*}$ is analogous to the transformation formula of Radu [37, Lemma 2.14] with respect to $\Gamma_{0}(N)^{*}$. The proof parallels that of Radu, and hence it is omitted.

Lemma 2.3. For a given partition function $a(n)$ as defined by (1.1), and for given integers $m$ and $t$, let $N$ be a positive integer satisfying the above conditions $1-\%$. For any $\gamma \in \Gamma_{1}(N)^{*}$, we have

$$
g_{m, t}(\gamma \tau)=(c \tau+d)^{\frac{1}{2} \sum_{\delta \mid M} r_{\delta}} e^{\pi i \zeta(\gamma)} \prod_{\delta \mid M} L(m \delta c, a)^{\left|r_{\delta}\right|} g_{m, t}(\tau),
$$


where

$$
L(c, a)= \begin{cases}\left(\frac{c}{a}\right), & \text { if } a>0, \\ \left(\frac{-c}{-a}\right), & \text { otherwise, }\end{cases}
$$

(-) is the Jacobi symbol,

$$
\zeta(\gamma)=\frac{a b\left(m^{2}-1\right) \alpha(t)}{12 m}+\frac{a b m}{12} \sum_{\delta \mid M} \delta r_{\delta}-\frac{a c m}{12} \sum_{\delta \mid M} \frac{r_{\delta}}{\delta}+\frac{\operatorname{sgn}(c)(a-1)}{4} \sum_{\delta \mid M} r_{\delta},
$$

and $\alpha(t)$ is defined as in the condition 5.

Next we derive a transformation formula for $F(\tau)$ under $\Gamma_{1}(N)^{*}$. Recall the notation of Schoeneberg [43]:

$$
\eta_{g, h}^{(s)}(\tau)=\alpha_{0}(h) e^{\pi i P_{2}\left(\frac{g}{\delta}\right) \tau} \prod_{\substack{m>0 \\ m \equiv g(\bmod \delta)}}\left(1-\zeta_{\delta}^{h} e^{\frac{2 \pi i \tau}{\delta} m}\right) \prod_{\substack{m>0 \\ m \equiv-g(\bmod \delta)}}\left(1-\zeta_{\delta}^{-h} e^{\frac{2 \pi i \tau}{\delta} m}\right),
$$

where $\zeta_{\delta}$ is a primitive $\delta$-th root of unity,

$$
\alpha_{0}(h)= \begin{cases}\left(1-\zeta_{\delta}^{-h}\right) e^{\pi i P_{1}\left(\frac{h}{\delta}\right)}, & \text { if } g \equiv 0(\bmod \delta) \text { and } h \neq 0(\bmod \delta), \\ 1, & \text { otherwise }\end{cases}
$$

the first Bernoulli function $P_{1}(x)$ is given by

$$
P_{1}(x)= \begin{cases}x-\lfloor x\rfloor-\frac{1}{2}, & \text { if } x \notin \mathbb{Z}, \\ 0, & \text { otherwise }\end{cases}
$$

and $\lfloor x\rfloor$ is the greatest integer less than or equal to $x$. Since

$$
\eta_{\delta, g}(\tau)=q^{\frac{\delta}{2} P_{2}\left(\frac{g}{\delta}\right)} \prod_{\substack{n>0 \\ n \equiv g(\bmod \delta)}}\left(1-q^{n}\right) \prod_{\substack{n>0 \\ n \equiv-g(\bmod \delta)}}\left(1-q^{n}\right),
$$

we have

$$
\eta_{\delta, g}(\tau)=\eta_{g, 0}^{(s)}(\delta \tau)
$$

Lemma 2.4. For a given partition function $a(n)$ as defined by (1.1), and for given integers $m$ and $t$, let $N$ be a positive integer satisfying the conditions $1-7$, and

$$
F(\tau)=\prod_{\delta \mid N} \eta^{a_{\delta}}(\delta \tau) \prod_{\substack{\delta \mid N \\ 0<g \leq\lfloor\delta / 2\rfloor}} \eta_{\delta, g}^{a_{\delta, g}}(\tau) g_{m, t}(\tau),
$$

where $a_{\delta}$ and $a_{\delta, g}$ are integers. Then for any $\gamma \in \Gamma_{1}(N)^{*}$,

$$
\begin{aligned}
F(\gamma \tau)=\prod_{\delta \mid N} L\left(\frac{c}{\delta}, a\right)^{a_{\delta}} \prod_{\delta \mid M} L(m \delta c, a)^{\left|r_{\delta}\right|} e^{\pi i(\nu(\gamma)+\xi(\gamma))} & \\
& \times(c \tau+d)^{\frac{1}{2}\left(\sum_{\delta \mid N} a_{\delta}+\sum_{\delta \mid M} r_{\delta}\right)} F(\tau),
\end{aligned}
$$


where

$$
\nu(\gamma)=\sum_{\substack{\delta \mid N \\ 0<g \leq\lfloor\delta / 2\rfloor}}\left(\frac{g}{\delta}-\frac{1}{2}\right)(a-1) a_{\delta, g}
$$

and

$$
\begin{aligned}
\xi(\gamma)= & \frac{a-1}{4} \operatorname{sgn}(c)\left(\sum_{\delta \mid N} a_{\delta}+\sum_{\delta \mid M} r_{\delta}\right) \\
& -a c\left(\sum_{\delta \mid N} \frac{a_{\delta}}{12 \delta}+\sum_{\substack{\delta \mid N \\
0<g \leq\lfloor\delta / 2\rfloor}} \frac{a_{\delta, g}}{6 \delta}+\sum_{\delta \mid M} \frac{m r_{\delta}}{12 \delta}\right) \\
& +a b\left(\sum_{\delta \mid N} \frac{\delta a_{\delta}}{12}+\sum_{\substack{\delta \mid N \\
0<g \leq \leq \delta / 2\rfloor}} \delta P_{2}\left(\frac{g}{\delta}\right) a_{\delta, g}+\sum_{\delta \mid M} \frac{m \delta r_{\delta}}{12}+\frac{\left(m^{2}-1\right) \alpha(t)}{12 m}\right) .
\end{aligned}
$$

Proof. For any $\gamma=\left(\begin{array}{ll}a & b \\ c & d\end{array}\right) \in \Gamma_{1}(N)^{*}$, we have

$$
F(\gamma \tau)=\prod_{\delta \mid N} \eta^{a_{\delta}}(\delta \gamma \tau) \prod_{\substack{\delta \mid N \\ 0<g \leq\lfloor\delta / 2\rfloor}} \eta_{\delta, g}^{a_{\delta, g}}(\gamma \tau) g_{m, t}(\gamma \tau) .
$$

For any $\delta \mid N$, let $\gamma_{\delta}^{\prime}=\left(\begin{array}{cc}a & \delta b \\ \frac{c}{\delta} & d\end{array}\right)$. Since $\gamma \in \Gamma_{1}(N)^{*}$, we have $N \mid c$ and so $\delta \mid c$ for any $\delta \mid N$. It follows that $\gamma_{\delta}^{\prime} \in \Gamma$. Thus (2.14) can be written as

$$
F(\gamma \tau)=\prod_{\delta \mid N} \eta^{a_{\delta}}\left(\gamma_{\delta}^{\prime}(\delta \tau)\right) \prod_{\substack{\delta \mid N \\ 0<g \leq\lfloor\delta / 2\rfloor}} \eta_{\delta, g}^{a_{\delta, g}}(\gamma \tau) g_{m, t}(\gamma \tau)
$$

The transformation formula of Newman [29, Lemma 2] states that for any $\gamma \in \Gamma$ with $a>0, c>0$ and $\operatorname{gcd}(a, 6)=1$,

$$
\eta(\gamma \tau)=\left(\frac{c}{a}\right) e^{-\frac{a \pi i}{12}(c-b-3)}(-i(c \tau+d))^{\frac{1}{2}} \eta(\tau) .
$$

Therefore, for any $\gamma \in \Gamma$ with $a c>0$ and $\operatorname{gcd}(a, 6)=1$, we have

$$
\eta(\gamma \tau)=L(c, a) e^{\pi i\left(\frac{a}{12}(-c+b)+\frac{a-1}{4} \operatorname{sgn}(c)\right)}(c \tau+d)^{\frac{1}{2}} \eta(\tau) .
$$

Since $\gamma \in \Gamma_{1}(N)^{*}$, we see that $\operatorname{gcd}(a, 6)=1$ and $a c>0$. Applying the transformation formula (2.16) to each $\gamma_{\delta}^{\prime}$, we deduce that

$$
\prod_{\delta \mid N} \eta^{a_{\delta}}\left(\gamma_{\delta}^{\prime}(\delta \tau)\right)=\prod_{\delta \mid N} L\left(\frac{c}{\delta}, a\right)^{a_{\delta}} e^{\pi i\left(\frac{a}{12}\left(-\frac{c}{\delta}+\delta b\right)+\frac{a-1}{4} \operatorname{sgn}(c)\right) a_{\delta}}(c \tau+d)^{\frac{a_{\delta}}{2}} \eta^{a_{\delta}}(\delta \tau) .
$$

Using the transformation formula of Robins [41, Theorem 2]:

$$
\eta_{\delta, g}(\gamma \tau)=e^{\pi i\left(\delta a b P_{2}\left(\frac{g}{\delta}\right)-\frac{a c}{6 \delta}+(a-1)\left(\frac{g}{\delta}-\frac{1}{2}\right)\right)} \eta_{\delta, g}(\tau),
$$


we find that

$$
\prod_{\substack{\delta \mid N \\ 0<g \leq\lfloor\delta / 2\rfloor}} \eta_{\delta, g}^{a_{\delta, g}}(\gamma \tau)=\prod_{\substack{\delta \mid N \\ 0<g \leq\lfloor\delta / 2\rfloor}} e^{\pi i\left(\delta a b P_{2}\left(\frac{g}{\delta}\right)-\frac{a c}{6 \delta}+(a-1)\left(\frac{g}{\delta}-\frac{1}{2}\right)\right) a_{\delta, g}} \eta_{\delta, g}^{a_{\delta, g}}(\tau) .
$$

Substituting the transformation formulas in (2.17), (2.18) and Lemma 2.3 into (2.15), we reach the transformation formula (2.11).

To prove Theorem 2.1, we need the Laurent expansions of $g_{m, t}(\gamma \tau)$ and $\phi(\gamma \tau)$ for $\gamma \in \Gamma$. Let us recall the two maps $p: \Gamma \times \mathbb{Z}_{m} \rightarrow \mathbb{Q}$ and $p: \Gamma \rightarrow \mathbb{Q}$ defined by Radu [37], namely, for $\gamma \in \Gamma$ and $\lambda \in \mathbb{Z}_{m}$,

$$
p(\gamma, \lambda)=\frac{1}{24} \sum_{\delta \mid M} \frac{\operatorname{gcd}^{2}(\delta(a+\kappa \lambda c), m c)}{\delta m} r_{\delta}
$$

and for $\gamma \in \Gamma$,

$$
p(\gamma)=\min \{p(\gamma, \lambda): \lambda=0,1, \ldots, m-1\} .
$$

The parabolic subgroup of $\Gamma$ is defined by

$$
\Gamma_{\infty}=\left\{ \pm\left(\begin{array}{ll}
1 & b \\
0 & 1
\end{array}\right): b \in \mathbb{Z}\right\}
$$

For any $\gamma \in \Gamma$, the $\left(\Gamma_{1}(N), \Gamma_{\infty}\right)$-double coset of $\gamma$ is given by

$$
\Gamma_{1}(N) \gamma \Gamma_{\infty}=\left\{\gamma_{N} \gamma \gamma_{\infty}: \gamma_{N} \in \Gamma_{1}(N), \gamma_{\infty} \in \Gamma_{\infty}\right\}
$$

Assume that $\Gamma$ has the following disjoint decomposition

$$
\Gamma=\bigcup_{i=1}^{\epsilon} \Gamma_{1}(N) \gamma_{i} \Gamma_{\infty}
$$

where $R=\left\{\gamma_{1}, \gamma_{2}, \ldots, \gamma_{\epsilon}\right\} \subseteq \Gamma$. Denote the set of $\left(\Gamma_{1}(N), \Gamma_{\infty}\right)$-double cosets in $\Gamma$ by $\Gamma_{1}(N) \backslash \Gamma / \Gamma_{\infty}$. Then $(2.21)$ can be written as

$$
\Gamma_{1}(N) \backslash \Gamma / \Gamma_{\infty}=\left\{\Gamma_{1}(N) \gamma \Gamma_{\infty}: \gamma \in R\right\} .
$$

We say that $R$ is a complete set of representatives of the double cosets $\Gamma_{1}(N) \backslash \Gamma / \Gamma_{\infty}$.

The following lemma gives a Laurent expansion of $g_{m, t}(\gamma \tau)$, and the proof is similar to that of Lemma 3.4 in Radu [37], and hence it is omitted.

Lemma 2.5. For a given partition function $a(n)$ as defined by (1.1), and for given integers $m$ and t, let $N$ be a positive integer satisfying the conditions $1-7$, and $R=\left\{\gamma_{1}, \gamma_{2}, \ldots, \gamma_{\epsilon}\right\}$ be a complete set of representatives of the double cosets $\Gamma_{1}(N) \backslash \Gamma / \Gamma_{\infty}$. For any $\gamma \in \Gamma$, assume that $\gamma \in \Gamma_{1}(N) \gamma_{i} \Gamma_{\infty}$ for some $1 \leq i \leq \epsilon$. Then there exists an integer $w$ and a Taylor series $h(q)$ in powers of $q^{\frac{1}{w}}$, such that

$$
g_{m, t}(\gamma \tau)=(c \tau+d)^{\frac{1}{2} \sum_{\delta \mid M} r_{\delta}} q^{p\left(\gamma_{i}\right)} h(q) .
$$

The following lemma gives a Laurent expansion of $\phi(\gamma \tau)$ for any $\gamma \in \Gamma$. 
Lemma 2.6. Let

$$
\phi(\tau)=\prod_{\delta \mid N} \eta^{a_{\delta}}(\delta \tau) \prod_{\substack{\delta \mid N \\ 0<g \leq\lfloor\delta / 2\rfloor}} \eta_{\delta, g}^{a_{\delta, g}}(\tau)
$$

where $a_{\delta}$ and $a_{\delta, g}$ are integers. For any $\gamma \in \Gamma$, there exists a positive integer $w$ and a Taylor series $h^{*}(q)$ in powers of $q^{\frac{1}{w}}$ such that

$$
\phi(\gamma \tau)=(c \tau+d)^{\frac{1}{2} \sum_{\delta \mid N} a_{\delta}} q^{p^{*}(\gamma)} h^{*}(q)
$$

where

$$
p^{*}(\gamma)=\frac{1}{24} \sum_{\delta \mid N} \frac{\operatorname{gcd}^{2}(\delta, c)}{\delta} a_{\delta}+\frac{1}{2} \sum_{\substack{\delta \mid N \\ 0<g \leq\lfloor\delta / 2\rfloor}} \frac{\operatorname{gcd}^{2}(\delta, c)}{\delta} P_{2}\left(\frac{a g}{\operatorname{gcd}(\delta, c)}\right) a_{\delta, g}
$$

Furthermore, for any $\gamma_{1} \in \Gamma$ and $\gamma_{2} \in \Gamma_{1}(N) \gamma_{1} \Gamma_{\infty}$, we have $p^{*}\left(\gamma_{1}\right)=p^{*}\left(\gamma_{2}\right)$.

Proof. For any $\gamma=\left(\begin{array}{ll}a & b \\ c & d\end{array}\right) \in \Gamma$, we have

$$
\phi(\gamma \tau)=\prod_{\delta \mid N} \eta^{a_{\delta}}(\delta \gamma \tau) \prod_{\substack{\delta \mid N \\ 0<g \leq\lfloor\delta / 2\rfloor}} \eta_{\delta, g}^{a_{\delta, g}}(\gamma \tau)
$$

It follows from (2.9) that

$$
\phi(\gamma \tau)=\prod_{\delta \mid N} \eta^{a_{\delta}}(\delta \gamma \tau) \prod_{\substack{\delta \mid N \\ 0<g \leq\lfloor\delta / 2\rfloor}} \eta_{g, 0}^{(s)^{a \delta, g}}(\delta \gamma \tau)
$$

Since $\operatorname{gcd}(a, c)=1$, for any $\delta \mid N$, there exist integers $x_{\delta}$ and $y_{\delta}$ such that

$$
\delta a x_{\delta}+c y_{\delta}=\operatorname{gcd}(\delta a, c)=\operatorname{gcd}(\delta, c),
$$

and hence

$$
\left(\begin{array}{cc}
\delta a & \delta b \\
c & d
\end{array}\right)=\left(\begin{array}{cc}
\frac{\delta a}{\operatorname{gcd}(\delta, c)} & -y_{\delta} \\
\frac{c}{\operatorname{gcd}(\delta, c)} & x_{\delta}
\end{array}\right)\left(\begin{array}{cc}
\operatorname{gcd}(\delta, c) & \delta b x_{\delta}+d y_{\delta} \\
0 & \frac{\delta}{\operatorname{gcd}(\delta, c)}
\end{array}\right)
$$

Set

$$
\gamma_{\delta}=\left(\begin{array}{cc}
\frac{\delta a}{\operatorname{gcd}(\delta, c)} & -y_{\delta} \\
\frac{c}{\operatorname{gcd}(\delta, c)} & x_{\delta}
\end{array}\right) \quad \text { and } \quad T_{\delta}=\left(\begin{array}{cc}
\operatorname{gcd}(\delta, c) & \delta b x_{\delta}+d y_{\delta} \\
0 & \frac{\delta}{\operatorname{gcd}(\delta, c)}
\end{array}\right) .
$$

Note that $\gamma_{\delta} \in \Gamma$. Combining (2.22) and (2.23), we deduce that

$$
\phi(\gamma \tau)=\prod_{\delta \mid N} \eta^{a_{\delta}}\left(\gamma_{\delta} T_{\delta} \tau\right) \prod_{\substack{\delta \mid N \\ 0<g \leq\lfloor\delta / 2\rfloor}} \eta_{g, 0}^{(s)^{a_{\delta, g}}}\left(\gamma_{\delta} T_{\delta} \tau\right)
$$

By the transformation law for $\eta(\tau)$ under $\Gamma\left[34\right.$, p. 145], namely, there exists a map $\varepsilon^{\prime}: \Gamma \rightarrow \mathbb{C}$ such that for any $\gamma \in \Gamma$,

$$
\eta(\gamma \tau)=\varepsilon^{\prime}(\gamma)(c \tau+d)^{\frac{1}{2}} \eta(\tau)
$$


and the transformation formula for $\eta_{g, h}^{(s)}(\tau)$ under $\Gamma$ in $[43$, p. $199(30)]$, namely, when $0<g<\delta$, there exists a map $\epsilon_{1}: \Gamma \rightarrow \mathbb{C}$ such that for any $\gamma \in \Gamma$,

$$
\eta_{g, h}^{(s)}(\gamma \tau)=\epsilon_{1}(\gamma) \eta_{g^{\prime}, h^{\prime}}^{(s)}(\tau)
$$

where $g^{\prime}=a g+c h, h^{\prime}=b g+d h$, it follows from (2.24) that there is a map $\varepsilon: \Gamma \rightarrow \mathbb{C}$ such that for any $\gamma \in \Gamma$,

$$
\phi(\gamma \tau)=\varepsilon(\gamma)(c \tau+d)^{\frac{1}{2} \sum_{\delta \mid N} a_{\delta}} \prod_{\delta \mid N} \eta^{a_{\delta}}\left(T_{\delta} \tau\right) \prod_{\substack{\delta \mid N \\ 0<g \leq\lfloor\delta / 2\rfloor}} \eta_{\frac{\delta a}{\operatorname{gcd}(\delta, c)} g,-y_{\delta} g}^{a_{\delta, g}}\left(T_{\delta} \tau\right)
$$

Substituting the $q$-expansions of the eta-function and the generalized eta-function into (2.25), we see that there exists a positive integer $w$ and a Taylor series $h^{*}(q)$ in powers of $q^{\frac{1}{w}}$ such that

$$
\phi(\gamma \tau)=(c \tau+d)^{\frac{1}{2} \sum_{\delta \mid N} a_{\delta}} q^{p^{*}(\gamma)} h^{*}(q) .
$$

Next we aim to show that $p^{*}\left(\gamma_{1}\right)=p^{*}\left(\gamma_{2}\right)$ for any $\gamma_{1} \in \Gamma$ and $\gamma_{2} \in \Gamma_{1}(N) \gamma_{1} \Gamma_{\infty}$. Under the assumption that $\gamma_{2} \in \Gamma_{1}(N) \gamma_{1} \Gamma_{\infty}$, there exist $\gamma_{3} \in \Gamma_{1}(N)$ and $\gamma_{4} \in \Gamma_{\infty}$ such that

$$
\gamma_{2}=\gamma_{3} \gamma_{1} \gamma_{4}
$$

Write

$$
\gamma_{1}=\left(\begin{array}{ll}
a_{1} & b_{1} \\
c_{1} & d_{1}
\end{array}\right), \quad \gamma_{2}=\left(\begin{array}{ll}
a_{2} & b_{2} \\
c_{2} & d_{2}
\end{array}\right), \quad \gamma_{3}=\left(\begin{array}{cc}
a_{3} & b_{3} \\
c_{3} & d_{3}
\end{array}\right), \quad \gamma_{4}=\left(\begin{array}{cc} 
\pm 1 & b_{4} \\
0 & \pm 1
\end{array}\right) .
$$

Owing to (2.26), we find that

$$
a_{2}= \pm\left(a_{1} a_{3}+b_{3} c_{1}\right)
$$

and

$$
c_{2}= \pm\left(a_{1} c_{3}+c_{1} d_{3}\right) .
$$

For any $\delta \mid N$, since $\gamma_{3} \in \Gamma_{1}(N)$, we see that $a_{3} \equiv 1(\bmod \delta), \delta \mid c_{3}$ and $\operatorname{gcd}\left(\delta, d_{3}\right)=1$. Using $(2.27)$, it can be verified that

$$
a_{2} g \equiv \pm a_{1} g \quad\left(\bmod \operatorname{gcd}\left(\delta, c_{1}\right)\right) .
$$

In view of (2.28), we obtain that

$$
\operatorname{gcd}\left(\delta, c_{2}\right)=\operatorname{gcd}\left(\delta, c_{1}\right) .
$$

Combining (2.29) and (2.30), we arrive at

$$
P_{2}\left(\frac{a_{1} g}{\operatorname{gcd}\left(\delta, c_{1}\right)}\right)=P_{2}\left(\frac{a_{2} g}{\operatorname{gcd}\left(\delta, c_{2}\right)}\right),
$$

here we have used the fact that $P_{2}(-\alpha)=P_{2}(\alpha)$ for any $\alpha \in \mathbb{R}$. Combining (2.30) and (2.31), we conclude that $p^{*}\left(\gamma_{1}\right)=p^{*}\left(\gamma_{2}\right)$, as claimed.

We are now ready to complete the proof of Theorem 2.1. 
Proof of Theorem 2.1. Assume that

$$
F(\tau)=\prod_{\delta \mid N} \eta^{a_{\delta}}(\delta \tau) \prod_{\substack{\delta \mid N \\ 0<g \leq\lfloor\delta / 2\rfloor}} \eta_{\delta, g}^{a_{\delta, g}}(\tau) g_{m, t}(\tau)
$$

is a modular function with respect to $\Gamma_{1}(N)$, where $a_{\delta}$ and $a_{\delta, g}$ are integers. We proceed to show that the conditions (1)-(4) are fulfilled by the integers $a_{\delta}$ and $a_{\delta, g}$.

Since $\Gamma_{1}(N)^{*} \subseteq \Gamma_{1}(N)$ and $F(\tau)$ is a modular function for $\Gamma_{1}(N)$, for any $\gamma \in \Gamma_{1}(N)^{*}$, we have

$$
F(\gamma \tau)=F(\tau) .
$$

To compute $F(\gamma \tau)$, we need the transformation formula for $F(\tau)$ under $\Gamma_{1}(N)^{*}$ as given in Lemma 2.4 , that is, for any $\gamma \in \Gamma_{1}(N)^{*}$,

$$
\begin{aligned}
F(\gamma \tau)=\prod_{\delta \mid N} L\left(\frac{c}{\delta}, a\right)^{a_{\delta}} \prod_{\delta \mid M} L( & m \delta c, a)^{\left|r_{\delta}\right|} e^{\pi i(\nu(\gamma)+\xi(\gamma))} \\
& \times(c \tau+d)^{\frac{1}{2}\left(\sum_{\delta \mid N} a_{\delta}+\sum_{\delta \mid M} r_{\delta}\right)} F(\tau),
\end{aligned}
$$

where $\nu(\gamma)$ and $\xi(\gamma)$ are defined in (2.12) and (2.13). Combining (2.33) and (2.34), we see that

$$
\sum_{\delta \mid N} a_{\delta}+\sum_{\delta \mid M} r_{\delta}=0
$$

thus (1) is satisfied. Consequently, $\xi(\gamma)$ reduces to

$$
\begin{aligned}
& -a c\left(\sum_{\delta \mid N} \frac{a_{\delta}}{12 \delta}+\sum_{\substack{\delta \mid N \\
0<g \leq\lfloor\delta / 2\rfloor}} \frac{a_{\delta, g}}{6 \delta}+\sum_{\delta \mid M} \frac{m r_{\delta}}{12 \delta}\right) \\
& +a b\left(\sum_{\delta \mid N} \frac{\delta a_{\delta}}{12}+\sum_{\substack{\delta \mid N \\
0<g \leq\lfloor\delta / 2\rfloor}} \delta P_{2}\left(\frac{g}{\delta}\right) a_{\delta, g}+\sum_{\delta \mid M} \frac{m \delta r_{\delta}}{12}+\frac{\left(m^{2}-1\right) \alpha(t)}{12 m}\right) .
\end{aligned}
$$

To prove (2), consider the matrix $\gamma=\left(\begin{array}{ll}1 & 0 \\ N & 1\end{array}\right) \in \Gamma_{1}(N)^{*}$. In this case, (2.34) becomes

$$
F(\gamma \tau)=e^{-\pi i N\left(\sum_{\delta \mid N} \frac{a_{\delta}}{12 \delta}+\sum_{\delta \mid N} \sum_{g=1}^{\lfloor\delta / 2\rfloor} \frac{a_{\delta, g}}{6 \delta}+\sum_{\delta \mid M} \frac{m r_{\delta}}{12 \delta}\right)} F(\tau) .
$$

Hence (2) follows from (2.33) and (2.35). Setting $\gamma=\left(\begin{array}{cc}1 & 1 \\ N & N+1\end{array}\right) \in \Gamma_{1}(N)^{*},(2.34)$ becomes

$$
F(\gamma \tau)=e^{\pi i\left(\sum_{\delta \mid N} \frac{\delta a_{\delta}}{12}+\sum_{\delta \mid N} \sum_{g=1}^{\lfloor\delta / 2\rfloor} \delta P_{2}\left(\frac{g}{\delta}\right) a_{\delta, g}+\sum_{\delta \mid M} \frac{m \delta r_{\delta}}{12}+\frac{\left(m^{2}-1\right) \alpha(t)}{12 m}\right)} F(\tau),
$$

which, together with (2.33), implies (3). Using the conditions (1)-(3), it can be checked that $\xi(\gamma) \equiv 0$ $(\bmod 2)$ for any $\gamma \in \Gamma_{1}(N)^{*}$. It follows that

$$
e^{\pi i \xi(\gamma)}=1,
$$

and so (2.34) reduces to

$$
F(\gamma \tau)=\prod_{\delta \mid N} L\left(\frac{c}{\delta}, a\right)^{a_{\delta}} \prod_{\delta \mid M} L(m \delta c, a)^{\left|r_{\delta}\right|} e^{\pi i \nu(\gamma)}(c \tau+d)^{\frac{1}{2}\left(\sum_{\delta \mid N} a_{\delta}+\sum_{\delta \mid M} r_{\delta}\right)} F(\tau) .
$$


By the definition of $L$, we find that for any $\delta \mid N$,

$$
L\left(\frac{c}{\delta}, a\right)=L(\delta c, a)=\left(\frac{\delta|c|}{|a|}\right),
$$

and for any $\delta \mid M$,

$$
L(m \delta c, a)=\left(\frac{m \delta|c|}{|a|}\right) .
$$

Hence (2.36) is equivalent to

$$
F(\gamma \tau)=\prod_{\delta \mid N}\left(\frac{\delta|c|}{|a|}\right)^{\left|a_{\delta}\right|} \prod_{\delta \mid M}\left(\frac{m \delta|c|}{|a|}\right)^{\left|r_{\delta}\right|} e^{\pi i \nu(\gamma)}(c \tau+d)^{\frac{1}{2}\left(\sum_{\delta \mid N} a_{\delta}+\sum_{\delta \mid M} r_{\delta}\right)} F(\tau) .
$$

In view of the condition (1), it is easily verified that

$$
(c \tau+d)^{\frac{1}{2}\left(\sum_{\delta \mid N} a_{\delta}+\sum_{\delta \mid M} r_{\delta}\right)}=1
$$

and

$$
\prod_{\delta \mid N}\left(\frac{|c|}{|a|}\right)^{\left|a_{\delta}\right|} \prod_{\delta \mid M}\left(\frac{|c|}{|a|}\right)^{\left|r_{\delta}\right|}=1
$$

Substituting (2.38) and (2.39) into (2.37) yields

$$
F(\gamma \tau)=\prod_{\delta \mid N}\left(\frac{\delta}{|a|}\right)^{\left|a_{\delta}\right|} \prod_{\delta \mid M}\left(\frac{m \delta}{|a|}\right)^{\left|r_{\delta}\right|} e^{\pi i \nu(\gamma)} F(\tau) .
$$

Comparing (2.33) with (2.40), we deduce that

$$
\prod_{\delta \mid N}\left(\frac{\delta}{|a|}\right)^{\left|a_{\delta}\right|} \prod_{\delta \mid M}\left(\frac{m \delta}{|a|}\right)^{\left|r_{\delta}\right|} e^{\pi i \nu(\gamma)}=1
$$

for all integers $a$ with $\operatorname{gcd}(a, 6)=1$ and $a \equiv 1(\bmod N)$. Invoking the interpretation of the Jacobi symbol, we conclude that (2.41) holds for all integers $0<a<12 N$ with $\operatorname{gcd}(a, 6)=1$ and $a \equiv 1$ $(\bmod N)$. This confirms $(4)$.

Conversely, assume that the integers $a_{\delta}, a_{\delta, g}(\delta \mid N, 0<g \leq\lfloor\delta / 2\rfloor)$ satisfy the conditions (1)-(4). We proceed to show that

$$
F(\tau)=\prod_{\delta \mid N} \eta^{a_{\delta}}(\delta \tau) \prod_{\substack{\delta \mid N \\ 0<g \leq\lfloor\delta / 2\rfloor}} \eta_{\delta, g}^{a_{\delta, g}}(\tau) g_{m, t}(\tau)
$$

is a modular function for $\Gamma_{1}(N)$. It is clear that $F(\tau)$ is holomorphic on $\mathbb{H}$.

Based on the conditions (1)-(3), it follows from Lemma 2.4 that the transformation formula (2.40) for $F(\tau)$ holds for any $\gamma \in \Gamma_{1}(N)^{*}$. Given the condition (4), we see that (2.41) holds for all integers $a$ with $\operatorname{gcd}(a, 6)=1$ and $a \equiv 1(\bmod N)$. Combining (2.40) and (2.41), we find that for any $\gamma \in \Gamma_{1}(N)^{*}$,

$$
F(\gamma \tau)=F(\tau) .
$$


In view of Lemma 2.2, we conclude that $F(\gamma \tau)=F(\tau)$ for any $\gamma \in \Gamma_{1}(N)$.

It remains to show that for any $\gamma \in \Gamma, F(\gamma \tau)$ has a Laurent expansion with a finite principal part in powers of $q^{\frac{1}{N}}$. Let $\gamma \in \Gamma$ and $R=\left\{\gamma_{1}, \gamma_{2}, \ldots, \gamma_{\epsilon}\right\}$ be a complete set of representatives of the double cosets $\Gamma_{1}(N) \backslash \Gamma / \Gamma_{\infty}$. By the decomposition of $\Gamma$ in (2.21), there exist an integer $1 \leq i \leq \epsilon$ and matrices $\gamma_{N} \in \Gamma_{1}(N), \gamma_{\infty} \in \Gamma_{\infty}$ such that $\gamma=\gamma_{N} \gamma_{i} \gamma_{\infty}$. By Lemma 2.5 and Lemma 2.6, there exist a positive integer $w$ and Taylor series $h(q)$ and $h^{*}(q)$ in powers of $q^{\frac{1}{w}}$ such that

$$
F(\gamma \tau)=(c \tau+d)^{\frac{1}{2}\left(\sum_{\delta \mid N} a_{\delta}+\sum_{\delta \mid M} r_{\delta}\right)} q^{p\left(\gamma_{i}\right)+p^{*}\left(\gamma_{i}\right)} h(q) h^{*}(q) .
$$

In view of the condition (1), (2.42) reduces to

$$
F(\gamma \tau)=q^{p\left(\gamma_{i}\right)+p^{*}\left(\gamma_{i}\right)} h(q) h^{*}(q),
$$

which implies that there exists a positive integer $k$ such that $F(\gamma \tau)$ has the Laurent expansion with a finite principal part in powers of $q^{\frac{1}{k}}$. Since we have shown that $F(\tau)$ is invariant under $\Gamma_{1}(N)$, by Lemma 1.14 in [48], we obtain that for any $\gamma \in \Gamma, F(\gamma \tau)$ is invariant under $\gamma^{-1} \Gamma_{1}(N) \gamma$. Notice that $\left(\begin{array}{cc}1 & N \\ 0 & 1\end{array}\right) \in \gamma^{-1} \Gamma_{1}(N) \gamma$. So $F(\gamma \tau)$ has period $N$, namely,

$$
F(\gamma(\tau+N))=F(\gamma \tau)
$$

Thus $F(\gamma \tau)$ has a Laurent expansion in powers of $q^{\frac{1}{N}}$. By (2.43), we see that this Laurent expansion has at most finitely many negative terms. So we reach the assertion that $F(\tau)$ is a modular function for $\Gamma_{1}(N)$.

Given a generating function of $a(n)$ as defined in (1.1) and integers $m$ and $t$, we can find an integer $N$ satisfying the conditions $1-7$. If we are lucky, we may use Theorem 2.1 to find integers $a_{\delta}, a_{\delta, g}$ $(\delta \mid N, 0<g \leq\lfloor\delta / 2\rfloor)$ satisfying the conditions (1)-(4), which lead to a generalized eta-quotient

$$
\phi(\tau)=\prod_{\delta \mid N} \eta^{a_{\delta}}(\delta \tau) \prod_{\substack{\delta \backslash N \\ 0<g \leq\lfloor\delta / 2\rfloor}} \eta_{\delta, g}^{a_{\delta, g}}(\tau)
$$

such that

$$
F(\tau)=\phi(\tau) g_{m, t}(\tau)
$$

is a modular function. It should be noted that such a modular function $F(\tau)$ may be not unique. To derive a Ramanujan-type identity for $a(m n+t)$, we aim to express $F(\tau)$ as a linear combination of generalized eta-quotients over $\mathbb{Q}$. To this end, we first investigate the behavior of $F(\tau)$ at each cusp of $\Gamma_{1}(N)$. Let us recall some terminology of modular functions, see, for example [12,48]. For $\gamma=\left(\begin{array}{ll}a & b \\ c & d\end{array}\right) \in \Gamma$, the width $w_{\gamma}$ of $\frac{a}{c}$ relative to $\Gamma_{1}(N)$ is the minimal positive integer $h$ such that

$$
\left(\begin{array}{ll}
1 & h \\
0 & 1
\end{array}\right) \in \gamma^{-1} \Gamma_{1}(N) \gamma
$$

Let $f(\tau)$ be a modular function for $\Gamma_{1}(N)$. It is known that $f(\gamma \tau)$ is invariant under $\gamma^{-1} \Gamma_{1}(N) \gamma$, see [48, Lemma 1.14]. So $f(\gamma \tau)$ has period $w_{\gamma}$, which implies that $f(\gamma \tau)$ has a Laurent expansion in powers of $q^{1 / w_{\gamma}}$. Since $f(\tau)$ is a modular function, this Laurent expansion has at most finitely many negative terms. Write

$$
f(\gamma \tau)=\sum_{n=-\infty}^{\infty} b_{n} q^{n / w_{\gamma}}
$$


where $b_{n}=0$ for almost all negative integers $n$. Let $n_{\gamma}$ be the smallest integer such that $b_{n_{\gamma}} \neq 0$. We call $n_{\gamma}$ the $\gamma$-order of $f$ at $\frac{a}{c}$, denoted by $\operatorname{ord}_{\gamma}(f)$. Denote the smallest exponent of $q$ on the right hand side of (2.45) by $v_{\gamma}$, so that

$$
\operatorname{ord}_{\gamma}(f)=v_{\gamma} w_{\gamma}
$$

Furthermore, the order of $f$ at the cusp $\frac{a}{c} \in \mathbb{Q} \cup\{\infty\}$ is defined by

$$
\operatorname{ord}_{a / c}(f)=\operatorname{ord}_{\gamma}(f)
$$

for some $\gamma \in \Gamma$ such that $\gamma \infty=\frac{a}{c}$. It is known that $\operatorname{ord}_{a / c}(f)$ is well-defined, see [12, p. 72].

The following theorem gives estimates of the orders of $F(\tau)$ at cusps of $\Gamma_{1}(N)$.

Theorem 2.7. For a given partition function $a(n)$ as defined by (1.1), and for given integers $m$ and $t$, let

$$
F(\tau)=\phi(\tau) g_{m, t}(\tau)
$$

where

$$
\phi(\tau)=\prod_{\delta \mid N} \eta^{a_{\delta}}(\delta \tau) \prod_{\substack{\delta \mid N \\ 0<g \leq\lfloor\delta / 2\rfloor}} \eta_{\delta, g}^{a_{\delta, g}}(\tau),
$$

$a_{\delta}$ and $a_{\delta, g}$ are integers. Assume that $F(\tau)$ is a modular function for $\Gamma_{1}(N)$. Let $\left\{s_{1}, s_{2}, \ldots, s_{\epsilon}\right\}$ be a complete set of inequivalent cusps of $\Gamma_{1}(N)$, and for each $1 \leq i \leq \epsilon$, let $\alpha_{i} \in \Gamma$ be such that $\alpha_{i} \infty=s_{i}$. Then

$$
\operatorname{ord}_{s_{i}}(F(\tau)) \geq w_{\alpha_{i}}\left(p\left(\alpha_{i}\right)+p^{*}\left(\alpha_{i}\right)\right),
$$

where $p(\gamma)$ is given by $(2.20)$ and $p^{*}(\gamma)$ is defined in Lemma 2.6.

To compute the right hand side of (2.48), we need the following formula due to Cho, Koo and Park [10]:

$$
w_{\gamma}= \begin{cases}1, & \text { if } N=4 \text { and } \operatorname{gcd}(c, 4)=2, \\ \frac{N}{\operatorname{gcd}(c, N)}, & \text { otherwise }\end{cases}
$$

where $\gamma=\left(\begin{array}{ll}a & b \\ c & d\end{array}\right) \in \Gamma$. For example, consider the modular function

$$
F(\tau)=q^{\frac{2}{5}} \frac{\eta(10 \tau) \eta_{10,5}^{9}(\tau)}{\eta_{10,4}^{8}(\tau)} \sum_{n=0}^{\infty} \bar{p}(5 n+2) q^{n}
$$

for $\Gamma_{1}(10)$ as given in (2.4). A complete set $\mathcal{S}(N)$ of inequivalent cusps of $\Gamma_{1}(N)$ has been found in [10, Corollary 4]. In particular, for $N=10$, we have

$$
\mathcal{S}(10)=\left\{0, \frac{1}{5}, \frac{1}{4}, \frac{3}{10}, \frac{1}{3}, \frac{3}{5}, \frac{1}{2}, \infty\right\} .
$$

Employing Theorem 2.7, we obtain the following lower bounds of the orders of $F(\tau)$ at cusps of $\Gamma_{1}(10)$ :

$$
\begin{aligned}
& \operatorname{ord}_{0}(F(\tau)) \geq-3, \operatorname{ord}_{1 / 5}(F(\tau)) \geq \frac{19}{5}, \operatorname{ord}_{1 / 4}(F(\tau)) \geq-2, \\
& \operatorname{ord}_{3 / 10}(F(\tau)) \geq-\frac{18}{5}, \operatorname{ord}_{1 / 3}(F(\tau)) \geq-3, \operatorname{ord}_{3 / 5}(F(\tau)) \geq \frac{27}{5}, \\
& \operatorname{ord}_{1 / 2}(F(\tau)) \geq-2, \operatorname{ord}_{\infty}(F(\tau)) \geq-\frac{2}{5} .
\end{aligned}
$$


Notice that $F(\tau)$ may have poles at some cusps not equivalent to infinity.

We are now ready to prove Theorem 2.7 .

Proof of Theorem 2.7. It is known that there exists a bijection from the set of all inequivalent cusps of $\Gamma_{1}(N)$ to the double coset space $\Gamma_{1}(N) \backslash \Gamma / \Gamma_{\infty}$, as given by

$$
\Gamma_{1}(N)(a / c) \mapsto \Gamma_{1}(N)\left(\begin{array}{ll}
a & b \\
c & d
\end{array}\right) \Gamma_{\infty},
$$

see [12, Proposition 3.8.5]. Since $\left\{s_{1}, s_{2}, \ldots, s_{\epsilon}\right\}$ is a complete set of inequivalent cusps of $\Gamma_{1}(N)$ and $\alpha_{i} \infty=s_{i}$ for $1 \leq i \leq \epsilon$, we see that $\left\{\alpha_{1}, \alpha_{2}, \ldots, \alpha_{\epsilon}\right\}$ is a complete set of representatives of $\Gamma_{1}(N) \backslash \Gamma / \Gamma_{\infty}$. Applying Lemma 2.5 with $\gamma_{i}=\alpha_{i}$, we find that there exists an integer $w_{1}$ and a Taylor series $h(q)$ in powers of $q^{\frac{1}{w_{1}}}$ such that

$$
g_{m, t}\left(\alpha_{i} \tau\right)=(c \tau+d)^{\frac{1}{2} \sum_{\delta \mid M} r_{\delta}} q^{p\left(\alpha_{i}\right)} h(q) .
$$

By Lemma 2.6, there exists a positive integer $w_{2}$ and a Taylor series $h^{*}(q)$ in powers of $q^{\frac{1}{w_{2}}}$, such that

$$
\phi\left(\alpha_{i} \tau\right)=(c \tau+d)^{\frac{1}{2} \sum_{\delta \mid N} a_{\delta}} q^{p^{*}\left(\alpha_{i}\right)} h^{*}(q) .
$$

Combining (2.51) and (2.52), we get

$$
F\left(\alpha_{i} \tau\right)=(c \tau+d)^{\frac{1}{2}\left(\sum_{\delta \mid N} a_{\delta}+\sum_{\delta \mid M} r_{\delta}\right)} q^{p\left(\alpha_{i}\right)+p^{*}\left(\alpha_{i}\right)} h(q) h^{*}(q) .
$$

Since $F(\tau)$ is a modular function for $\Gamma_{1}(N)$, using the condition (1) in Theorem 2.1, (2.53) reduces to

$$
F\left(\alpha_{i} \tau\right)=q^{p\left(\alpha_{i}\right)+p^{*}\left(\alpha_{i}\right)} h(q) h^{*}(q) .
$$

Let $v_{\alpha_{i}}$ denote the smallest exponent of $q$ on the right hand side of (2.54). The relation $\operatorname{ord}_{\gamma}(f)=v_{\gamma} w_{\gamma}$ as given in (2.46) yields

$$
v_{\alpha_{i}}=\frac{\operatorname{ord}_{\alpha_{i}}(F(\tau))}{w_{\alpha_{i}}}
$$

Since $h(q)$ and $h^{*}(q)$ are Taylor series, it follows from (2.54) that

$$
v_{\alpha_{i}} \geq p\left(\alpha_{i}\right)+p^{*}\left(\alpha_{i}\right) .
$$

Combining (2.55) and (2.56), we conclude that

$$
\operatorname{ord}_{\alpha_{i}}(F(\tau)) \geq w_{\alpha_{i}}\left(p\left(\alpha_{i}\right)+p^{*}\left(\alpha_{i}\right)\right) .
$$

By the definition (2.47), we have

$$
\operatorname{ord}_{s_{i}}(F(\tau))=\operatorname{ord}_{\alpha_{i}}(F(\tau)) .
$$

Thus the estimate (2.48) follows from (2.57) and (2.58). 


\section{Sketch of the Algorithm}

In this section, we give a sketch of our algorithm. Given a generating function of $a(n)$ as defined in (1.1) and integers $m$ and $t$, we can find an integer $N$ satisfying the conditions $1-7$. Assume that we have found a generalized eta-quotient $\phi(\tau)$ such that

$$
F(\tau)=\phi(\tau) g_{m, t}(\tau)
$$

is a modular function for $\Gamma_{1}(N)$. To derive an expression of $F(\tau)$, we consider a class of modular functions: the set of generalized eta-quotients which are modular functions for $\Gamma_{1}(N)$ with poles only at infinity, denoted by $G E^{\infty}(N)$. Note that the notation $E^{\infty}(N)$ is used by Radu [35] to denote the set of modular eta-quotients with poles only at infinity for $\Gamma_{0}(N)$. Our goal is to derive an expression of $F(\tau)$ in terms of the generators of $G E^{\infty}(N)$. Then we are led to a Ramanujan-type identity for $a(m n+t)$.

Our algorithm consists of the following steps:

Step 1. Use Theorem 2.1 to find a generalized eta-quotient $\phi(\tau)$ for which $F(\tau)$ in (3.1) is a modular function for $\Gamma_{1}(N)$.

Step 2. Find a finite set $\left\{z_{1}, z_{2}, \ldots, z_{k}\right\}$ of generators of $G E^{\infty}(N)$ by utilizing a formula of Robins and the theory of Diophantine inequalities.

Step 3. Let $\left\langle G E^{\infty}(N)\right\rangle_{\mathbb{Q}}$ be the vector space over $\mathbb{Q}$ generated by generalized eta-quotients in $G E^{\infty}(N)$. Employ the Algorithm AB of Radu for $\Gamma_{1}(N)$ on $\left\{z_{1}, z_{2}, \ldots, z_{k}\right\}$ to generate a modular function $z$ and a $\mathbb{Q}[z]$-module basis $1, e_{1}, \ldots, e_{w}$ of $\left\langle G E^{\infty}(N)\right\rangle_{\mathbb{Q}}$.

Step 4. Find a generalized eta-quotient $h$ in terms of generators of $G E^{\infty}(N)$ for which the modular function $h F$ has a pole only at infinity. Theorem 2.7 can be used to compute the lower bounds of the orders of $h F$ at all cusps of $\Gamma_{1}(N)$.

Step 5. Determine whether $h F$ is in $\left\langle G E^{\infty}(N)\right\rangle_{\mathbb{Q}}$ by applying the Algorithm MW of Radu to $h F, z$ and $1, e_{1}, \ldots, e_{w}$. If this goal can be achieved, then $F$ can be expressed as a linear combination of generalized eta-quotients over $\mathbb{Q}$.

For example, let us consider the overpartition function $\bar{p}(5 n+2)$. In Sect. 2., we found $N=10$ satisfies the conditions $1-7$.

Step 1. As shown in (2.4),

$$
F(\tau)=q^{\frac{2}{5}} \frac{\eta(10 \tau) \eta_{10,5}^{9}(\tau)}{\eta_{10,4}^{8}(\tau)} \sum_{n=0}^{\infty} \bar{p}(5 n+2) q^{n}
$$

is a modular function with respect to $\Gamma_{1}(10)$. 
Step 2. We obtain the following generators of $G E^{\infty}(10)$ :

$$
\begin{aligned}
& z=\frac{\eta(\tau) \eta(5 \tau)}{\eta_{5,1}^{2}(\tau) \eta^{2}(10 \tau) \eta_{10,1}(\tau)}, \quad z_{1}=\frac{\eta^{2}(2 \tau) \eta(5 \tau) \eta_{5,1}^{2}(\tau)}{\eta(\tau) \eta^{2}(10 \tau) \eta_{10,1}^{4}(\tau)} \\
& z_{2}=\frac{\eta^{3}(5 \tau) \eta_{5,1}^{4}(\tau)}{\eta(\tau) \eta(2 \tau) \eta(10 \tau) \eta_{10,1}^{3}(\tau)}, \quad z_{3}=\frac{\eta(\tau) \eta_{5,1}^{2}(\tau) \eta^{2}(10 \tau)}{\eta^{2}(2 \tau) \eta(5 \tau) \eta_{10,1}^{4}(\tau)} \\
& z_{4}=\frac{\eta^{4}(\tau) \eta_{5,1}^{2}(\tau)}{\eta^{3}(2 \tau) \eta(10 \tau) \eta_{10,1}^{4}(\tau)}
\end{aligned}
$$

Step 3. Applying the Algorithm AB of Radu to $\left\{z, z_{1}, z_{2}, z_{3}, z_{4}\right\}$, we find that 1 is a $z$-module basis of $\left\langle G E^{\infty}(10)\right\rangle_{\mathbb{Q}}$. Thus

$$
\left\langle G E^{\infty}(10)\right\rangle_{\mathbb{Q}}=\langle 1\rangle_{\mathbb{Q}[z]}
$$

Step 4. We obtain that

$$
h=\frac{z_{1}^{2} z_{3}^{3} z_{4}^{3}}{z^{6} z_{2}^{4}}=\frac{\eta^{11}(\tau) \eta_{5,1}^{12}(\tau) \eta^{15}(10 \tau)}{\eta^{7}(2 \tau) \eta^{19}(5 \tau) \eta_{10,1}^{14}(\tau)},
$$

for which $h F$ has a pole only at infinity.

Step 5. Applying Radu's Algorithm MW to $h F, z$ and 1, we see that $h F \in\left\langle G E^{\infty}(10)\right\rangle_{\mathbb{Q}}$ and

$$
h F=4 z^{3}+4 z^{2}-32 z+32 .
$$

The relation (3.5) can be restated as the following theorem. The implementations of the above steps will be described in the subsequent sections.

Theorem 3.1. We have

$$
y \sum_{n=0}^{\infty} \bar{p}(5 n+2) q^{n}=4 z^{3}+4 z^{2}-32 z+32,
$$

where

$$
\begin{aligned}
& y=\frac{(q ; q)_{\infty}^{11}\left(q^{10} ; q^{10}\right)_{\infty}^{16}\left(q, q^{4} ; q^{5}\right)_{\infty}^{12}\left(q^{5} ; q^{10}\right)_{\infty}^{18}}{q^{3}\left(q^{2} ; q^{2}\right)_{\infty}^{7}\left(q^{5} ; q^{5}\right)_{\infty}^{19}\left(q, q^{9} ; q^{10}\right)_{\infty}^{14}\left(q^{4}, q^{6} ; q^{10}\right)_{\infty}^{8}} \\
& z=\frac{(q ; q)_{\infty}\left(q^{5} ; q^{5}\right)_{\infty}}{q\left(q, q^{4} ; q^{5}\right)_{\infty}^{2}\left(q^{10} ; q^{10}\right)_{\infty}^{2}\left(q, q^{9} ; q^{10}\right)_{\infty}}
\end{aligned}
$$

\section{Generators of $G E^{\infty}(N)$}

In this section, we show how to implement Step 1 as in the sketch of the previous section, that is, finding a finite set of generators of $G E^{\infty}(N)$.

In light of the symmetry

$$
\eta_{\delta, g}(\tau)=\eta_{\delta, \delta-g}(\tau),
$$


for any $\delta>0$ and $\lfloor\delta / 2\rfloor<g \leq \delta$, we may rewrite the generalized eta-quotient $h(\tau)$ in $G E^{\infty}(N)$ in the following form

$$
\prod_{\substack{\delta \leq N \\ 0 \leq g \leq\lfloor\delta / 2\rfloor}} \eta_{\delta, g}^{a_{\delta, g}}(\tau)
$$

where

$$
a_{\delta, g} \in \begin{cases}\frac{1}{2} \mathbb{Z}, & \text { if } g=0 \text { or } g=\frac{\delta}{2}, \\ \mathbb{Z}, & \text { otherwise. }\end{cases}
$$

Throughout this section, we assume that the generalized eta-quotients are of the form (4.1).

To find a set of generators of $G E^{\infty}(N)$, we first give a characterization of generalized eta-quotients $h(\tau)$ in $G E^{\infty}(N)$, which involves the orders of $h(\tau)$ at all cusps of $\Gamma_{1}(N)$. For any cusp $s$ of $\Gamma_{1}(N)$, in order to apply a formula of Robins [41, Theorem 4] to compute the order of $h(\tau)$ at a cusp $s$, we need to find a cusp of the form $\frac{\lambda}{\mu \varepsilon}$ that is equivalent to $s$, where $\varepsilon \mid N$ and

$$
\operatorname{gcd}(\lambda, N)=\operatorname{gcd}(\lambda, \mu)=\operatorname{gcd}(\mu, N)=1 .
$$

The existence of such a cusp in the above form is ensured by Corollary 4 of Cho, Koo and Park [10].

The following theorem gives a characterization of generalized eta-quotients in $G E^{\infty}(N)$.

Theorem 4.1. Let

$$
\mathcal{S}(N)=\left\{s_{1}, s_{2}, \ldots, s_{\epsilon}\right\}
$$

be a complete set of inequivalent cusps of $\Gamma_{1}(N)$ and $s_{\epsilon}=\infty$. Assume that for any $1 \leq i \leq \epsilon, s_{i}$ is equivalent to $\frac{\lambda_{i}}{\mu_{i} \varepsilon_{i}}$, where $\varepsilon_{i} \mid N$ and

$$
\operatorname{gcd}\left(\lambda_{i}, N\right)=\operatorname{gcd}\left(\lambda_{i}, \mu_{i}\right)=\operatorname{gcd}\left(\mu_{i}, N\right)=1 .
$$

Then a generalized eta-quotient $h(\tau)$ in the form of (4.1) is in $G E^{\infty}(N)$ if and only if the following conditions hold:

$$
\left\{\begin{array}{l}
\sum_{\delta \mid N} a_{\delta, 0}=0 \\
\frac{N}{2} \sum_{\substack{\delta \mid N \\
0 \leq g \leq\lfloor\delta / 2\rfloor}} \frac{\operatorname{gcd}^{2}\left(\delta, \varepsilon_{1}\right)}{\delta \varepsilon_{1}} P_{2}\left(\frac{\lambda_{1} g}{\operatorname{gcd}\left(\delta, \varepsilon_{1}\right)}\right) a_{\delta, g} \in \mathbb{N}, \\
\vdots \\
\frac{N}{2} \sum_{\substack{\delta \mid N \\
0 \leq \delta \leq\lfloor\delta / 2\rfloor}} \frac{\operatorname{gcd}^{2}\left(\delta, \varepsilon_{\epsilon-1}\right)}{\delta \varepsilon_{\epsilon-1}} P_{2}\left(\frac{\lambda_{\epsilon-1} g}{\operatorname{gcd}\left(\delta, \varepsilon_{\epsilon-1}\right)}\right) a_{\delta, g} \in \mathbb{N}, \\
\frac{N}{2} \sum_{\substack{\delta \backslash N \\
0 \leq g \leq\lfloor\delta / 2\rfloor}} \frac{\operatorname{gcd}^{2}\left(\delta, \varepsilon_{\epsilon}\right)}{\delta \varepsilon_{\epsilon}} P_{2}\left(\frac{\lambda_{\epsilon} g}{\operatorname{gcd}\left(\delta, \varepsilon_{\epsilon}\right)}\right) a_{\delta, g} \in \mathbb{Z} .
\end{array}\right.
$$

Proof. Assume that the generalized eta-quotient $h(\tau)$ as given by (4.1) is in $G E^{\infty}(N)$. By the transformation formula of Schoeneberg [43, p. $199(30)]$ for $\eta_{g, h}^{(s)}(\tau)$, we have

$$
\sum_{\delta \mid N} a_{\delta, 0}=0
$$


and so the first condition in (4.5) is satisfied. To show that the remaining conditions in (4.5) are satisfied, we proceed to compute the order of $h(\tau)$ at each cusp in $\mathcal{S}(N)$. Since $h(\tau) \in G E^{\infty}(N)$, for all $1 \leq i \leq \epsilon-1$,

$$
\operatorname{ord}_{s_{i}}(h(\tau)) \in \mathbb{N}
$$

and

$$
\operatorname{ord}_{s_{\epsilon}}(h(\tau)) \in \mathbb{Z} \text {. }
$$

For any $1 \leq i \leq \epsilon$, since $s_{i}$ is equivalent to $\frac{\lambda_{i}}{\mu_{i} \varepsilon_{i}}$, we get

$$
\operatorname{ord}_{s_{i}}(h(\tau))=\operatorname{ord}_{\lambda_{i} / \mu_{i} \varepsilon_{i}}(h(\tau)) .
$$

Using the formula of Robins [41, Theorem 4] for the order of $h(\tau)$ at the cusp $\lambda_{i} / \mu_{i} \varepsilon_{i}$, namely,

$$
\operatorname{ord}_{\lambda_{i} / \mu_{i} \varepsilon_{i}}(h(\tau))=\frac{N}{2} \sum_{\substack{\delta \mid N \\ 0 \leq g \leq\lfloor\delta / 2\rfloor}} \frac{\operatorname{gcd}^{2}\left(\delta, \varepsilon_{i}\right)}{\delta \varepsilon_{i}} P_{2}\left(\frac{\lambda_{i} g}{\operatorname{gcd}\left(\delta, \varepsilon_{i}\right)}\right) a_{\delta, g},
$$

we find that

$$
\operatorname{ord}_{s_{i}}(h(\tau))=\frac{N}{2} \sum_{\substack{\delta \backslash N \\ 0 \leq g \leq\lfloor\delta / 2\rfloor}} \frac{\operatorname{gcd}^{2}\left(\delta, \varepsilon_{i}\right)}{\delta \varepsilon_{i}} P_{2}\left(\frac{\lambda_{i} g}{\operatorname{gcd}\left(\delta, \varepsilon_{i}\right)}\right) a_{\delta, g} .
$$

For $1 \leq i \leq \epsilon-1$, combining (4.7) and (4.9), we obtain that

$$
\frac{N}{2} \sum_{\substack{\delta \mid N \\ 0 \leq g \leq \downarrow \delta / 2\rfloor}} \frac{\operatorname{gcd}^{2}\left(\delta, \varepsilon_{i}\right)}{\delta \varepsilon_{i}} P_{2}\left(\frac{\lambda_{i} g}{\operatorname{gcd}\left(\delta, \varepsilon_{i}\right)}\right) a_{\delta, g} \in \mathbb{N} .
$$

Setting $i=\epsilon$ in (4.9), it follows from (4.8) that

$$
\frac{N}{2} \sum_{\substack{\delta \mid N \\ 0 \leq g \leq\llcorner\delta / 2\rfloor}} \frac{\operatorname{gcd}^{2}\left(\delta, \varepsilon_{\epsilon}\right)}{\delta \varepsilon_{\epsilon}} P_{2}\left(\frac{\lambda_{\epsilon} g}{\operatorname{gcd}\left(\delta, \varepsilon_{\epsilon}\right)}\right) a_{\delta, g} \in \mathbb{Z} .
$$

Combining (4.6), (4.10) and (4.11), we are led to (4.5).

Conversely, assume that the conditions in (4.5) are satisfied. From (4.5) and (4.9), we see that

$$
\operatorname{ord}_{0}(h(\tau)) \in \mathbb{Z} \quad \text { and } \quad \operatorname{ord}_{\infty}(h(\tau)) \in \mathbb{Z} .
$$

The first condition of (4.5) says that

$$
\sum_{\delta \mid N} a_{\delta, 0}=0
$$

Robins [41] showed that if a generalized eta-quotient $h(\tau)$ satisfies (4.12) and (4.13), then for any $\gamma \in \Gamma_{1}(N)$

$$
h(\gamma \tau)=h(\tau) .
$$

By (4.9) and the conditions in (4.5), we see that for any $s \in \mathcal{S}(N) \backslash\{\infty\}$,

$$
\operatorname{ord}_{s}(h(\tau)) \in \mathbb{N} .
$$

Combining (4.14) and (4.15), we conclude that $h(\tau) \in G E^{\infty}(N)$. 
Based on the above theorem, the generalized eta-quotients in $G E^{\infty}(N)$ are determined by the solutions of (4.5). Next we show that (4.5) can be solved by transforming the conditions in (4.5) to a system of Diophantine inequalities, so that we can obtain a finite set of generators of $G E^{\infty}(N)$.

Set

$$
y_{i}=\frac{N}{2} \sum_{\substack{\delta \mid N \\ 0 \leq g \leq\lfloor\delta / 2\rfloor}} \frac{\operatorname{gcd}^{2}\left(\delta, \varepsilon_{i}\right)}{\delta \varepsilon_{i}} P_{2}\left(\frac{\lambda_{i} g}{\operatorname{gcd}\left(\delta, \varepsilon_{i}\right)}\right) a_{\delta, g}
$$

for $1 \leq i \leq \epsilon$. It follows from (4.5) that $y_{i} \in \mathbb{N}$ for $1 \leq i \leq \epsilon-1$ and $y_{\epsilon} \in \mathbb{Z}$. Let

$$
\chi_{\delta}(g)= \begin{cases}2, & \text { if } g=0 \text { or } g=\frac{\delta}{2} \\ 1, & \text { otherwise }\end{cases}
$$

and $a_{\delta, g}^{\prime}=\chi_{\delta}(g) a_{\delta, g}$ for any $\delta \mid N$ and $0 \leq g \leq\lfloor\delta / 2\rfloor$. By (4.2), it can be easily checked that each $a_{\delta, g}^{\prime}$ is an integer. Then by Theorem 4.1, $h(\tau) \in G E^{\infty}(N)$ if and only if $a_{\delta, g}^{\prime}(\delta \mid N, 0 \leq g \leq\lfloor\delta / 2\rfloor)$ and $y_{i}$ $(1 \leq i \leq \epsilon)$ is an integer solution of the following Diophantine inequalities:

$$
\left\{\begin{array}{l}
\sum_{\delta \mid N} a_{\delta, 0}^{\prime}=0 \\
\frac{N}{2} \sum_{\substack{\delta \mid N \\
0 \leq g \leq l \delta / 2\rfloor}} \frac{\operatorname{gcd}^{2}\left(\delta, \varepsilon_{1}\right)}{\delta \varepsilon_{1}} P_{2}\left(\frac{\lambda_{1} g}{\operatorname{gcd}\left(\delta, \varepsilon_{1}\right)}\right) \frac{a_{\delta, g}^{\prime}}{\chi \delta(g)}-y_{1}=0 \\
\vdots \\
\frac{N}{2} \sum_{\substack{\delta \mid N \\
0 \leq g \leq l \delta / 2\rfloor}} \frac{\operatorname{gcd}^{2}\left(\delta, \varepsilon_{\epsilon-1}\right)}{\delta \varepsilon_{\epsilon-1}} P_{2}\left(\frac{\lambda_{\epsilon-1} g}{\operatorname{gcd}\left(\delta, \varepsilon_{\epsilon-1}\right)}\right) \frac{a_{\delta, g}^{\prime}}{\chi_{\delta}(g)}-y_{\epsilon-1}=0 \\
\frac{N}{2} \sum_{\substack{\delta \mid N \\
0 \leq g \leq l \delta / 2\rfloor}} \frac{\operatorname{gcd}^{2}\left(\delta, \varepsilon_{\epsilon}\right)}{\delta \varepsilon_{\epsilon}} P_{2}\left(\frac{\lambda_{\epsilon} g}{\operatorname{gcd}\left(\delta, \varepsilon_{\epsilon}\right)}\right) \frac{a_{\delta, g}^{\prime}}{\chi_{\delta}(g)}-y_{\epsilon}=0 \\
y_{1} \geq 0, \underbrace{\prime} \\
\quad \vdots \\
y_{\epsilon-1} \geq 0
\end{array}\right.
$$

Notice that different cusps may have the same order for $h(\tau)$, there may exist redundant relations in above system of relations. More precisely, if for two cusps $s_{i}, s_{j} \in \mathcal{S}(N) \backslash\{\infty\}$,

$$
\operatorname{ord}_{s_{i}}(h(\tau))=\operatorname{ord}_{s_{j}}(h(\tau)),
$$

then we may ignore the relations contributed by $s_{j}$. We now assume that after the elimination of redundant relations, the remaining relations are still in the same form as in (4.16). It is known that there exist integral vectors $\alpha_{1}, \ldots, \alpha_{k}$ such that the set of integer solutions of (4.16) is given by

$$
\left\{u_{1} \alpha_{1}+\cdots+u_{k} \alpha_{k}: u_{1}, \ldots, u_{k} \in \mathbb{N}\right\},
$$

see [44, p. 234], which implies that $G E^{\infty}(N)$ has a finite set of generators $z_{1}, \ldots, z_{k}$. One can use the package 4ti2 [1] in SAGE to find such a set of integral vectors $\alpha_{1}, \ldots, \alpha_{k}$.

Let us consider the case $N=10$ as an example. Notice that for any generalized eta-quotient $h(\tau)$,

$$
\operatorname{ord}_{1 / 4}(h(\tau))=\operatorname{ord}_{1 / 2}(h(\tau))
$$


and

$$
\operatorname{ord}_{0}(h(\tau))=\operatorname{ord}_{1 / 3}(h(\tau)) .
$$

By (4.16), we obtain the following Diophantine inequalities after eliminating the relations contributed by the cusps $1 / 2$ and $1 / 3$ :

$$
\left\{\begin{array}{l}
a_{1,0}^{\prime}+a_{2,0}^{\prime}+a_{5,0}^{\prime}+a_{10,0}^{\prime}=0, \\
\frac{5 a_{1,0}^{\prime}}{12}+\frac{5 a_{2,0}^{\prime}}{24}+\frac{5 a_{2,1}^{\prime}}{24}+\frac{a_{5,0}^{\prime}}{12}+\frac{a_{5,1}}{6}+\frac{a_{5,2}}{6} \\
\quad+\frac{a_{10,0}^{\prime}}{24}+\frac{a_{10,1}}{12}+\frac{a_{10,2}}{12}+\frac{a_{10,3}}{12}+\frac{a_{10,4}}{12}+\frac{a_{10,5}^{\prime}}{24}-y_{1}=0, \\
\quad \vdots \\
\frac{5 a_{1,0}^{\prime}}{24}+\frac{5 a_{2,0}^{\prime}}{12}-\frac{5 a_{2,1}^{\prime}}{24}+\frac{a_{5,0}^{\prime}}{24}+\frac{a_{5,1}^{\prime}}{12}+\frac{a_{5,2}^{\prime}}{12} \\
\quad+\frac{a_{10,0}^{\prime}}{12}-\frac{a_{10,1}^{\prime}}{12}+\frac{a_{10,2}^{\prime}}{6}-\frac{a_{10,3}^{\prime}}{12}+\frac{a_{10,4}^{\prime}}{6}-\frac{a_{10,5}^{\prime}}{24}-y_{5}=0, \\
\frac{a_{1,0}^{\prime}}{24}+\frac{a_{2,0}^{\prime}}{12}-\frac{a_{2,1}^{\prime}}{24}+\frac{5 a_{5,0}^{\prime}}{24}+\frac{a_{5,1}^{\prime}}{60}-\frac{11 a_{5,2}^{\prime}}{60} \\
\quad+\frac{5 a_{10,0}^{\prime}}{12}+\frac{23 a_{10,1}^{\prime}}{60}+\frac{a_{10,2}^{\prime}}{30}-\frac{13 a_{10,3}^{\prime}}{60}-\frac{11 a_{10,4}^{\prime}}{30}-\frac{5 a_{10,5}^{\prime}}{24}-y_{6}=0 \\
y_{1} \geq 0 \\
\quad \vdots \\
y_{5} \geq 0
\end{array}\right.
$$

Each solution $\left(a_{1,0}^{\prime}, \ldots, a_{10,5}^{\prime}, y_{1}, \ldots, y_{6}\right)$ of $(4.17)$ can be expressed as

$$
\sum_{i=1}^{5} c_{i} \alpha_{i}+\sum_{i=1}^{6} d_{i} \beta_{i}
$$

where $c_{1}, \ldots, c_{5}$ are nonnegative integers, $d_{1}, \ldots, d_{6}$ are integers and

$$
\begin{aligned}
& \alpha_{1}=(-1,2,0,1,2,0,-2,-4,0,0,0,0,0,1,0,0,0,-2), \\
& \alpha_{2}=(-1,-1,0,3,4,0,-1,-3,0,0,0,0,0,0,1,0,0,-1), \\
& \alpha_{3}=(1,-2,0,-1,2,0,2,-4,0,0,0,0,0,0,0,0,1,-1), \\
& \alpha_{4}=(1,0,0,1,-2,0,-2,-1,0,0,0,0,0,0,0,1,0,-1), \\
& \alpha_{5}=(4,-3,0,0,2,0,-1,-4,0,0,0,0,1,0,0,0,0,-2), \\
& \beta_{1}=(0,0,0,-1,0,0,1,0,0,0,0,1,0,0,0,0,0,0), \\
& \beta_{2}=(-1,1,1,0,0,0,0,0,0,0,0,0,0,0,0,0,0,0), \\
& \beta_{3}=(-1,0,0,1,1,1,0,0,0,0,0,0,0,0,0,0,0,0), \\
& \beta_{4}=(0,-1,0,0,1,0,1,-1,1,0,0,0,0,0,0,0,0,0), \\
& \beta_{5}=(-1,1,0,1,0,0,-1,1,0,1,0,0,0,0,0,0,0,0),
\end{aligned}
$$




$$
\beta_{6}=(0,0,0,0,-1,0,0,1,0,0,1,0,0,0,0,0,0,0) .
$$

Since $a_{\delta, g}=a_{\delta, g}^{\prime} / \chi_{\delta}(g)$, we obtain eleven generalized eta-quotients. It can be checked that the generalized eta-quotients corresponding to $\beta_{1}, \ldots, \beta_{6}$ are equal to 1 . For example, the generalized eta-quotient corresponding to $\beta_{1}$ is given by

$$
h(\tau)=\frac{\eta_{10,0}^{\frac{1}{2}}(\tau) \eta_{10,5}^{\frac{1}{2}}(\tau)}{\eta_{5,0}^{\frac{1}{2}}(\tau)} .
$$

Invoking (1.12), namely,

$$
\eta_{\delta, 0}(\tau)=\eta^{2}(\delta \tau) \quad \text { and } \quad \eta_{\delta, \frac{\delta}{2}}(\tau)=\frac{\eta^{2}\left(\frac{\delta}{2} \tau\right)}{\eta^{2}(\delta \tau)}
$$

we obtain that $h(\tau)=1$. The generalized eta-quotients corresponding to $\alpha_{1}, \ldots, \alpha_{5}$ are the generators $z_{1}, z_{2}, z_{3}, z, z_{4}$ as given in (3.2).

\section{Radu's Algorithm AB}

In the previous section, it was shown that $G E^{\infty}(N)$ admits a finite set of generators $z_{1}, \ldots, z_{k}$. Radu [37] developed the Algorithm AB to produce a module basis of $\left\langle E^{\infty}(N)\right\rangle_{\mathbb{Q}}$, based on a finite set of generators of $E^{\infty}(N)$. In this section, we demonstrate how to apply Radu's Algorithm AB to a finite set of generators of $G E^{\infty}(N)$ to derive a modular function $z$ and a module basis $1, e_{1}, \ldots, e_{w}$ of the $\mathbb{Q}[z]$-module $\left\langle G E^{\infty}(N)\right\rangle_{\mathbb{Q}}$.

We first give an overview of Radu's Algorithm AB. Given modular functions $z_{1}, \ldots, z_{k}$ for $\Gamma_{0}(N)$ with poles only at infinity, Radu's Algorithm $\mathrm{AB}$ aims to produce a modular function $z \in \mathbb{Q}\left[z_{1}, \ldots, z_{k}\right]$ and a $z$-reduced sequence $e_{1}, \ldots, e_{w} \in \mathbb{Q}\left[z_{1}, \ldots, z_{k}\right]$ such that

$$
\mathbb{Q}\left[z_{1}, \ldots, z_{k}\right]=\mathbb{Q}[z]+\mathbb{Q}[z] e_{1}+\cdots+\mathbb{Q}[z] e_{w} .
$$

The condition on a $z$-reduced sequence ensures that $1, e_{1}, \ldots, e_{w}$ form a $\mathbb{Q}[z]$-module basis of $\mathbb{Q}\left[z_{1}, \ldots, z_{k}\right]$. The right hand side of $(5.1)$ is denoted by $\left\langle 1, e_{1}, \ldots, e_{w}\right\rangle_{\mathbb{Q}[z]}$.

Let $\left\langle E^{\infty}(N)\right\rangle_{\mathbb{Q}}$ denote the vector space over $\mathbb{Q}$ generated by $E^{\infty}(N)$. As pointed out by Radu [35], $\left\langle E^{\infty}(N)\right\rangle_{\mathbb{Q}}$ does not have a finite basis as a vector space over $\mathbb{Q}$, but it has a finite basis when considered as a $\mathbb{Q}[z]$-module for some $z$ in $\left\langle E^{\infty}(N)\right\rangle_{\mathbb{Q}}$. To obtain such a modular function $z$ and a $\mathbb{Q}[z]$-module basis, Radu applied the Algorithm $\mathrm{AB}$ to the generators $z_{1}, \ldots, z_{k}$ of $E^{\infty}(N)$, then obtained a $z$ module basis $1, e_{1}, \ldots, e_{w}$ of the $\mathbb{Q}[z]$-module $\left\langle E^{\infty}(N)\right\rangle_{\mathbb{Q}}$ for some $z \in\left\langle E^{\infty}(N)\right\rangle_{\mathbb{Q}}$.

As will be seen, Radu's Algorithm $\mathrm{AB}$ can be adapted to $\Gamma_{1}(N)$. The output of Algorithm AB consists of a modular function $z \in \mathbb{Q}\left[z_{1}, \ldots, z_{k}\right]$ and a $z$-reduced sequence $e_{1}, \ldots, e_{w}$. The output of the Algorithm AB will be carried over to the Algorithm MC and the Algorithm MW, which require the input of a $z$-reduced sequence. Thus, for the purpose of this paper, we do not need to elaborate on the definition of a $z$-reduced sequence, which can be found in [35].

It is known that if $f$ is a modular function for $\Gamma_{0}(N)$ such that $\operatorname{ord}_{a / c}(f) \geq 0$ for every cusp $a / c$ of $\Gamma_{0}(N)$, then $f$ is a constant, see Newman [28, Section, Proof of Lemma 3], Knopp [25, Chapter 2, Theorem 7], and Radu [35, Lemma 5]. Notice that this assertion also holds for $\Gamma_{1}(N)$. Thus the Algorithm $\mathrm{AB}$ applies to modular functions with poles only at infinity for $\Gamma_{1}(N)$. It is worth mentioning 
that the Algorithm AB is based on the algorithms MC, VB and MB, which are also valid for modular functions with poles only at infinity for $\Gamma_{1}(N)$. Since the Algorithm MW of Radu is a refinement of the Algorithm MC, it also works for $\Gamma_{1}(N)$.

We proceed to find a modular function $z$ and a module basis of $\mathbb{Q}[z]$-module $\left\langle G E^{\infty}(N)\right\rangle_{\mathbb{Q}}$. Let $\left\{z_{1}, \ldots, z_{k}\right\}$ be a finite set of generators of $G E^{\infty}(N)$. Note that

$$
\left\langle G E^{\infty}(N)\right\rangle_{\mathbb{Q}}=\mathbb{Q}\left[z_{1}, \ldots, z_{k}\right] .
$$

Applying the Algorithm $\mathrm{AB}$ to $z_{1}, z_{2}, \ldots, z_{k}$, we obtain a modular function $z \in\left\langle G E^{\infty}(N)\right\rangle_{\mathbb{Q}}$ and a $z$-reduced sequence $e_{1}, \ldots, e_{w} \in\left\langle G E^{\infty}(N)\right\rangle_{\mathbb{Q}}$ such that

$$
\mathbb{Q}\left[z_{1}, \ldots, z_{k}\right]=\left\langle 1, e_{1}, \ldots, e_{w}\right\rangle_{\mathbb{Q}[z]} .
$$

Combining (5.2) and (5.3), we find that

$$
\left\langle G E^{\infty}(N)\right\rangle_{\mathbb{Q}}=\left\langle 1, e_{1}, \ldots, e_{w}\right\rangle_{\mathbb{Q}[z]} .
$$

Using the property that $e_{1}, e_{2}, \ldots, e_{w}$ form a $z$-reduced sequence, we deduce that $1, e_{1}, \ldots, e_{w}$ constitute a $\mathbb{Q}[z]$-module basis of $\left\langle G E^{\infty}(N)\right\rangle_{\mathbb{Q}}$.

For example, applying the Algorithm $\mathrm{AB}$ for $\Gamma_{1}(N)$ to the generators $z, z_{1}, z_{2}, z_{3}, z_{4}$ of $G E^{\infty}(10)$ given by (3.2), we obtain that

$$
\left\langle G E^{\infty}(10)\right\rangle_{\mathbb{Q}}=\mathbb{Q}[z]
$$

\section{Finding a Generalized Eta-Quotient}

In this section, we present an implementation of Step 4 in the algorithm outlined in Sect. 3.. Assume that $\left\{z_{1}, z_{2}, \ldots, z_{k}\right\}$ is a set of generators of $G E^{\infty}(N)$ and $F(\tau)$ is a modular function for $\Gamma_{1}(N)$ as given in (3.1). Our objective is to find a generalized eta-quotient $h(\tau)$ of the form

$$
h(\tau)=\prod_{j=1}^{k} z_{j}^{t_{j}},
$$

such that the modular function $h F$ has a pole only at infinity, that is, for any cusp $s \neq \infty$,

$$
\operatorname{ord}_{s}(h F) \geq 0
$$

where $t_{j}$ are integers. To find the integers $t_{j}$ for which the relation (6.2) holds, we shall establish a system of linear inequalities any solution of which leads to a desired generalized eta-quotient $h$. The linear inequalities are derived by the lower bounds of $\operatorname{ord}_{s}(h F)$ for all cusps $s \neq \infty$.

Now we utilize Theorem 2.7 to obtain the lower bound of $\operatorname{ord}_{s}(h F)$. Let

$$
\mathcal{S}(N)=\left\{s_{1}, s_{2}, \ldots, s_{\epsilon}\right\}
$$

be a complete set of inequivalent cusps of $\Gamma_{1}(N)$ and $s_{\epsilon}=\infty$. For any $1 \leq i \leq \epsilon$ and $1 \leq j \leq k$, denote $\operatorname{ord}_{s_{i}} z_{j}$ by $b_{i j}$. By the definition (6.1), we have for each cusp $s_{i}$,

$$
\operatorname{ord}_{s_{i}}(h F)=\sum_{j=1}^{k} t_{j} b_{i j}+\operatorname{ord}_{s_{i}}(F) .
$$


By Theorem 2.7, we see that for any $1 \leq i \leq \epsilon$,

$$
\operatorname{ord}_{s_{i}}(F(\tau)) \geq d_{i}
$$

where

$$
d_{i}=w_{\alpha_{i}}\left(p\left(\alpha_{i}\right)+p^{*}\left(\alpha_{i}\right)\right)
$$

and $\alpha_{i}$ is defined in Theorem 2.7. Combining (6.3) and (6.4), we get

$$
\operatorname{ord}_{s_{i}}(h F) \geq \sum_{j=1}^{k} t_{j} b_{i j}+d_{i} .
$$

Consider the Diophantine inequalities

$$
\left\{\begin{array}{c}
\sum_{j=1}^{k} t_{j} b_{1 j}+d_{1}>-1 \\
\vdots \\
\sum_{j=1}^{k} t_{j} b_{(\epsilon-1) j}+d_{\epsilon-1}>-1 .
\end{array}\right.
$$

Now, if we can find integers $t_{1}, \ldots, t_{k}$ such that (6.6) holds, then (6.5) implies that the generalized eta-quotient $h(\tau)$ determined by $z_{1}, z_{2}, \ldots, z_{k}$ and $t_{1}, t_{2}, \ldots, t_{k}$ satisfies (6.2). Hence we deduce that any integer solution of (6.6) leads to a generalized eta-quotient $h(\tau)$ such that $h F$ has a pole only at infinity.

We note that different generalized eta-quotients $h$ may lead to different expressions for $F$. In order to get a relatively simple expression for $F$, we impose a further condition that the order of $h F$ at infinity is as large as possible. While we cannot rigorously describe what a simple expression means, intuitively speaking, the above condition appears to play a role in getting a relatively simple expression for $F$. Next we state how to find such a generalized eta-quotient $h(\tau)$.

It is known that there exist integral vectors $\alpha_{1}, \ldots, \alpha_{w}, \beta_{1}, \ldots, \beta_{l}$ such that the set of integer solutions of (6.6) is given by

$$
\left\{\alpha_{i}+v_{1} \beta_{1}+\cdots+v_{l} \beta_{l}: 1 \leq i \leq w \text { and } v_{1}, \ldots, v_{l} \in \mathbb{N}\right\},
$$

see $[44$, p. 234].

The following theorem shows how to find a generalized eta-quotient $h$ such that $\operatorname{ord}_{\infty}(h F)$ attains the maximum value among all the $h$ satisfying (6.6).

Theorem 6.1. For $1 \leq i \leq w$, let

$$
\alpha_{i}=\left(\alpha_{i 1}, \alpha_{i 2}, \ldots, \alpha_{i k}\right),
$$

as given in (6.7). Let $h_{i}$ be the generalized eta-quotient determined by $z_{1}, z_{2}, \ldots, z_{k}$ and $\alpha_{i}$, that is

$$
h_{i}(\tau)=\prod_{j=1}^{k} z_{j}^{\alpha_{i j}}
$$

Assume that

$$
\operatorname{ord}_{\infty}\left(h_{1} F\right) \geq \operatorname{ord}_{\infty}\left(h_{i} F\right)
$$


for $2 \leq i \leq w$. For any integer solution $\mu=\left(\mu_{1}, \mu_{2}, \ldots, \mu_{k}\right)$ of (6.6), let $g$ be the generalized eta-quotient

$$
g(\tau)=\prod_{j=1}^{k} z_{j}^{\mu_{j}} .
$$

Then we have

$$
\operatorname{ord}_{\infty}\left(h_{1} F\right) \geq \operatorname{ord}_{\infty}(g F) .
$$

Proof. By (6.7), there exist an integer $1 \leq i \leq w$, and nonnegative integers $v_{1}, \ldots, v_{l}$ such that

$$
\mu=\alpha_{i}+v_{1} \beta_{1}+\cdots+v_{l} \beta_{l} .
$$

For $1 \leq j \leq l$, let

$$
\beta_{j}=\left(\beta_{j 1}, \beta_{j 2}, \ldots, \beta_{j k}\right) .
$$

and let $f_{j}$ be the generalized eta-quotient defined by

$$
f_{j}(\tau)=\prod_{i=1}^{k} z_{i}^{\beta_{j i}} .
$$

Combining (6.8), (6.11) and (6.12), we obtain that

$$
g(\tau)=h_{i} \prod_{j=1}^{l} f_{j}^{v_{j}} .
$$

Thus,

$$
\operatorname{ord}_{\infty}(g F)=\operatorname{ord}_{\infty}\left(h_{i} F\right)+\sum_{j=1}^{l} v_{j} \operatorname{ord}_{\infty}\left(f_{j}\right) .
$$

Under the condition (6.9), it follows from (6.13) that

$$
\operatorname{ord}_{\infty}(g F) \leq \operatorname{ord}_{\infty}\left(h_{1} F\right)+\sum_{j=1}^{l} v_{j} \operatorname{ord}_{\infty}\left(f_{j}\right)
$$

We claim that for each $1 \leq j \leq l$,

$$
\operatorname{ord}_{\infty}\left(f_{j}\right) \leq 0
$$

There are two cases.

Case 1. If $f_{j}(\tau)$ is a constant, then $\operatorname{ord}_{\infty}\left(f_{j}\right)=0$.

Case 2. If $f_{j}(\tau)$ is not a constant, we shall show that $\operatorname{ord}_{\infty}\left(f_{j}\right)<0$. Assume to the contrary that $\operatorname{ord}_{\infty}\left(f_{j}\right) \geq 0$. Since $f_{j}(\tau)$ is not a constant, there exists a cusp $s \neq \infty$ such that $\operatorname{ord}_{s}\left(f_{j}\right)<0$. By the assumption (6.2), we have $\operatorname{ord}_{s}\left(h_{1} F\right) \geq 0$. Let $d=\operatorname{ord}_{s}\left(h_{1} F\right)$. By (6.7), we see that $\alpha_{1}+(d+1) \beta_{j}$ is a solution of (6.6). It follows that the generalized eta-quotient $f_{j}^{d+1} h_{1}$ satisfies (6.2), and so

$$
\operatorname{ord}_{s}\left(f_{j}^{d+1} h_{1} F\right) \geq 0 .
$$


However, since $\operatorname{ord}_{s}\left(f_{j}\right)<0$, we have

$$
\operatorname{ord}_{s}\left(f_{j}^{d+1} h_{1} F\right)=(d+1) \operatorname{ord}_{s}\left(f_{j}\right)+d<0,
$$

which contradicts (6.16). Thus we deduce that $\operatorname{ord}_{\infty}\left(f_{j}\right)<0$, as claimed. Combining the above two cases, we find that (6.15) holds for each $1 \leq j \leq l$. In view of (6.14), we conclude that

$$
\operatorname{ord}_{\infty}(g F) \leq \operatorname{ord}_{\infty}\left(h_{1} F\right)
$$

and this completes the proof.

For the overpartition function $\bar{p}(5 n+2)$, we have found a modular function $F(\tau)$ for $\Gamma_{1}(10)$ as given in (2.4). For the generators $z, z_{1}, z_{2}, z_{3}, z_{4}$ of $G E^{\infty}(10)$ as given in (3.2), we obtain the following system of linear inequalities (6.6):

$$
\left\{\begin{array}{l}
t_{5}-3>-1, \\
t_{3}+\frac{19}{5}>-1 \\
t_{2}-2>-1 \\
t_{4}-\frac{18}{5}>-1 \\
t_{1}+\frac{27}{5}>-1
\end{array}\right.
$$

Each integer solution $\left(t_{1}, t_{2}, t_{3}, t_{4}, t_{5}\right)$ of $(6.18)$ can be expressed as

$$
\alpha_{1}+\sum_{i=1}^{5} v_{i} \beta_{i},
$$

where $v_{1}, \ldots, v_{5}$ are nonnegative integers, and

$$
\begin{aligned}
& \alpha_{1}=(-6,2,-4,3,3), \\
& \beta_{1}=(1,0,0,0,0), \\
& \beta_{2}=(0,1,0,0,0), \\
& \beta_{3}=(0,0,1,0,0), \\
& \beta_{4}=(0,0,0,1,0), \\
& \beta_{5}=(0,0,0,0,1) .
\end{aligned}
$$

The generalized eta-quotient corresponding to $\alpha_{1}$ is

$$
h=\frac{z_{1}^{2} z_{3}^{3} z_{4}^{3}}{z^{6} z_{2}^{4}}=\frac{\eta^{11}(\tau) \eta_{5,1}^{12}(\tau) \eta^{15}(10 \tau)}{\eta^{7}(2 \tau) \eta^{19}(5 \tau) \eta_{10,1}^{14}(\tau)}
$$

and $h F$ has a pole only at infinity. Consider a different solution $\mu=\alpha_{1}+2 \beta_{2}=(6,4,-4,3,3)$ of (6.18), we get a generalized eta-quotient

$$
h^{\prime}=\frac{z_{1}^{4} z_{3}^{3} z_{4}^{3}}{z^{6} z_{2}^{4}}=\frac{\eta^{9}(\tau) \eta_{5,1}^{16}(\tau) \eta^{11}(10 \tau)}{\eta^{3}(2 \tau) \eta^{17}(5 \tau) \eta_{10,1}^{22}(\tau)}
$$

and $h^{\prime} F$ has a pole only at infinity. The orders of $h F$ and $h^{\prime} F$ at infinity are -3 and -7 , respectively. As will be seen in the next section, the Ramanujan-type identity derived from $h F$ takes a simpler form than that derived from $h^{\prime} F$. 


\section{Ramanujan-Type Identities}

Given a partition function $a(n)$ as defined by (1.1), and integers $m$ and $t$, let

$$
F(\tau)=\phi(\tau) g_{m, t}(\tau)
$$

be a modular function as given in (3.1), where $\phi(\tau)$ is a generalized eta-quotient of the form (2.2), and

$$
g_{m, t}(\tau)=q^{\frac{t-\ell}{m}} \sum_{n=0}^{\infty} a(m n+t) q^{n},
$$

as given in (2.1).

Assume that we have found a generalized eta-quotient $h(\tau)$ such that $h F$ has a pole only at infinity. In Sect. 5., we derived a modular function $z \in\left\langle G E^{\infty}(N)\right\rangle_{\mathbb{Q}}$ and a $z$-reduced sequence $e_{1}, \ldots, e_{w}$ such that

$$
\left\langle G E^{\infty}(N)\right\rangle_{\mathbb{Q}}=\mathbb{Q}[z]+\mathbb{Q}[z] e_{1}+\cdots+\mathbb{Q}[z] e_{w} .
$$

In this section, we aim to derive an expression for $h F$ in terms of $z$ and the module basis $1, e_{1}, \ldots, e_{w}$. This leads to a Ramanujan-type identity for $a(m n+t)$.

We first adapt Radu's Algorithm MC, original designed for $\Gamma_{0}(N)$, to $\Gamma_{1}(N)$, and apply it to $h F, z$ and $e_{1}, \ldots, e_{w}$ to determine whether $h F$ belongs to $\left\langle G E^{\infty}(N)\right\rangle_{\mathbb{Q}}$. By Radu [35, Lemma 5], the Algorithm MC requires the non-positive parts of the $q$-expansion of $h F$, and finite parts of the $q$-expansions of $z$, and $e_{1}, \ldots, e_{w}$. More precisely, by (7.1), the non-positive parts of the $q$-expansion of $h F$ can be computed via the generating function (1.1) of $a(n)$ and the $q$-expansions of $h(\tau)$ and $\phi(\tau)$. If the algorithm confirms that $h F \in\left\langle G E^{\infty}(N)\right\rangle_{\mathbb{Q}}$, then we may utilize the $\Gamma_{1}(N)$ version of Algorithm MW to express $h F$ as

$$
h F=p_{0}(z)+p_{1}(z) e_{1}+\cdots+p_{w}(z) e_{w},
$$

where $p_{i}(z) \in \mathbb{Q}[z]$ for $0 \leq i \leq w$.

To this end, we first utilize the Radu's Algorithm $\mathrm{MC}$ for $\Gamma_{1}(N)$ to determine whether $h F$ belongs to $\left\langle G E^{\infty}(N)\right\rangle_{\mathbb{Q}}$. Once we have confirmed that $h F \in\left\langle G E^{\infty}(N)\right\rangle_{\mathbb{Q}}$, we may utilize the Algorithm MW of Radu for $\Gamma_{1}(N)$ to derive a Ramanujan-type identity for $a(m n+t)$.

We now give an algorithmic derivation of the Ramanujan-type identity for $\bar{p}(5 n+2)$, as stated in Theorem 3.1.

For $F, z$ and $h$ given in (2.4), (3.2) and (6.20), we have

$$
\begin{gathered}
h F=\frac{4}{q^{3}}+\frac{28}{q^{2}}+\frac{56}{q}+140+O(q), \\
z=\frac{1}{q}+2+2 q+q^{2}+O\left(q^{3}\right) .
\end{gathered}
$$

Applying Radu's Algorithm MC to $h F$ and $z$, we deduce that

$$
h F \in\left\langle G E^{\infty}(10)\right\rangle_{\mathbb{Q}} .
$$


With the input $h F$ and $z$, the Algorithm MW yields

$$
h F=4 z^{3}+4 z^{2}-32 z+32 .
$$

Substituting $F, z$ and $h$ into (7.3), we obtain the Ramanujan-type identity in Theorem 3.1. However, if we take $h^{\prime}$ as given in (6.21), then we get

$$
h^{\prime} F=4 z^{7}-4 z^{6}-44 z^{5}+100 z^{4}-20 z^{3}-92 z^{2}+32 z+32 .
$$

In the same vain, we obtain a Ramanujan-type identity for $\bar{p}(5 n+3)$.

Theorem 7.1. We have

$$
y \sum_{n=0}^{\infty} \bar{p}(5 n+3) q^{n}=8 z^{3}-12 z^{2}+16 z-16,
$$

where $z$ is given in Theorem 3.1 and

$$
y=\frac{(q ; q)_{\infty}^{12}\left(q^{5} ; q^{5}\right)_{\infty}^{12}\left(q, q^{9} ; q^{10}\right)_{\infty}^{2}\left(q^{4}, q^{6} ; q^{10}\right)_{\infty}^{8}}{q^{3}\left(q^{2} ; q^{2}\right)_{\infty}^{7}\left(q, q^{4} ; q^{5}\right)_{\infty}^{6}\left(q^{10} ; q^{10}\right)_{\infty}^{16}\left(q^{5} ; q^{10}\right)_{\infty}^{14}}
$$

Notice that Theorem 3.1 and Theorem 7.1 can be considered as witness identities for the following congruences of Hirschhorn and Sellers [24]:

$$
\begin{array}{ll}
\bar{p}(5 n+2) \equiv 0 & (\bmod 4), \\
\bar{p}(5 n+3) \equiv 0 & (\bmod 4) .
\end{array}
$$

\section{A Witness Identity for $p(11 n+6)$}

In this section, we demonstrate how our algorithm gives rise to a witness identity for $p(11 n+6)$. We begin with an overview of the witness identities due to Bilgici and Ekin [8], Radu [35] and Hemmecke [20]. Bilgici and Ekin [8] used the method of Kolberg to deduce the generating functions of $p(11 n+t)$ for all $0 \leq t \leq 10$. In particular, they obtained the following witness identity:

$$
\begin{array}{rl}
\sum_{n=0}^{\infty} p(11 n+6) q^{n}=11 & x\left(-x_{1}^{3} x_{4}-x_{2}^{3} x_{5}-x_{4}^{3} x_{2}-x_{3}^{3} x_{1}-x_{5}^{3} x_{3}-14 x_{1}^{2} x_{4}\right. \\
& -14 x_{2}^{2} x_{5}-14 x_{4}^{2} x_{2}-14 x_{3}^{2} x_{1}-14 x_{5}^{2} x_{3}-29 x_{1} x_{4} \\
& \left.-29 x_{2} x_{5}-29 x_{2} x_{4}-29 x_{1} x_{3}-29 x_{3} x_{5}+106\right),
\end{array}
$$

where

$$
\begin{aligned}
x & =\frac{q^{4}\left(q^{11} ; q^{11}\right)_{\infty}^{11}}{(q ; q)_{\infty}^{12}}, \\
x_{1} & =-\frac{\left(q^{4}, q^{7} ; q^{11}\right)_{\infty}^{2}\left(q, q^{10} ; q^{11}\right)_{\infty}}{\left(q^{2}, q^{9} ; q^{11}\right)_{\infty}^{2}\left(q^{5}, q^{6} ; q^{11}\right)_{\infty}} \\
x_{2} & =-\frac{\left(q^{2}, q^{9} ; q^{11}\right)_{\infty}^{2}\left(q^{5}, q^{6} ; q^{11}\right)_{\infty}}{q\left(q, q^{10} ; q^{11}\right)_{\infty}^{2}\left(q^{3}, q^{8} ; q^{11}\right)_{\infty}}
\end{aligned}
$$




$$
\begin{aligned}
& x_{3}=\frac{q^{2}\left(q, q^{10} ; q^{11}\right)_{\infty}^{2}\left(q^{3}, q^{8} ; q^{11}\right)_{\infty}}{\left(q^{4}, q^{7} ; q^{11}\right)_{\infty}\left(q^{5}, q^{6} ; q^{11}\right)_{\infty}^{2}}, \\
& x_{4}=\frac{\left(q^{4}, q^{7} ; q^{11}\right)_{\infty}\left(q^{5}, q^{6} ; q^{11}\right)_{\infty}^{2}}{q\left(q^{2}, q^{9} ; q^{11}\right)_{\infty}\left(q^{3}, q^{8} ; q^{11}\right)_{\infty}^{2}}, \\
& x_{5}=-\frac{\left(q^{2}, q^{9} ; q^{11}\right)_{\infty}\left(q^{3}, q^{8} ; q^{11}\right)_{\infty}^{2}}{\left(q^{4}, q^{7} ; q^{11}\right)_{\infty}^{2}\left(q, q^{10} ; q^{11}\right)_{\infty}} .
\end{aligned}
$$

Using the Ramanujan-Kolberg algorithm, Radu [35] derived a witness identity for $p(11 n+6)$. A set $\left\{M_{1}, M_{2}, \ldots, M_{7}\right\}$ of generators of $E^{\infty}(22)$ can be found in [35]. For example,

$$
M_{1}=\frac{\eta^{7}(\tau) \eta^{3}(11 \tau)}{\eta^{3}(2 \tau) \eta^{7}(22 \tau)}
$$

Let

$$
F=\frac{(q ; q)_{\infty}^{10}\left(q^{2} ; q^{2}\right)_{\infty}^{2}\left(q^{11} ; q^{11}\right)_{\infty}^{11}}{q^{14}\left(q^{22} ; q^{22}\right)_{\infty}^{22}} \sum_{n=0}^{\infty} p(11 n+6) q^{n}
$$

Radu showed that

$$
\begin{aligned}
F=11 & \left(98 t^{4}+1263 t^{3}+2877 t^{2}+1019 t-1997\right) \\
+ & 11 z_{1}\left(17 t^{2}+490 t^{2}+54 t-871\right) \\
+ & 11 z_{2}\left(t^{3}+251 t^{2}+488 t-614\right),
\end{aligned}
$$

where

$$
\begin{aligned}
t & =\frac{3}{88} M_{1}+\frac{1}{11} M_{2}-\frac{1}{8} M_{4}, \\
z_{1} & =-\frac{5}{88} M_{1}+\frac{2}{11} M_{2}-\frac{1}{8} M_{4}-3, \\
z_{2} & =\frac{1}{44} M_{1}-\frac{3}{11} M_{2}+\frac{5}{4} M_{4} .
\end{aligned}
$$

Noting that $\left(1-q^{n}\right)^{11} \equiv 1-q^{11 n}(\bmod 11)$ and $\left(1-q^{n}\right)^{8} \equiv\left(1-q^{2 n}\right)^{4}(\bmod 8)$, we see that $(8.2)$ implies the Ramanujan congruence for $p(11 n+6)$. Hemmecke [20] generalized Radu's algorithm and derived the following witness identity:

$$
\begin{aligned}
F=11^{2} \cdot 3068 M_{7}+11^{2} \cdot\left(3 M_{1}+4236\right) M_{6} \\
+11 \cdot\left(285 M_{1}+11 \cdot 5972\right) M_{5}+11\left(1867 M_{1}+11 \cdot 2476\right) M_{2} \\
\quad-\frac{11}{8}\left(M_{1}^{3}+1011 M_{1}^{2}+11 \cdot 6588 M_{1}+11^{2} \cdot 10880\right) \\
+\frac{11}{8}\left(M_{1}^{2}+11 \cdot 4497 M_{1}+11^{2} \cdot 3156\right) M_{4} .
\end{aligned}
$$

We are now ready to give an algorithmic derivation of the identity for $p(11 n+6)$ as stated in Theorem 1.1. 
Proof of Theorem 1.1. Notice that $N=11$ satisfies all the conditions 1-7. We proceed with the following steps.

Step 1. By Theorem 2.1, we find that

$$
F(\tau)=q\left(q^{11} ; q^{11}\right)_{\infty} \sum_{n=0}^{\infty} p(11 n+6) q^{n}
$$

is a modular function for $\Gamma_{1}(11)$.

Step 2. Solving the system of Diophantine inequalities (4.16) for $N=11$, we obtain a set of 27 generators of $G E^{\infty}(11)$ including $z$ and $e$ as given in (1.8) and (1.9).

Step 3. Applying Radu's Algorithm AB, we deduce that

$$
\left\langle G E^{\infty}(11)\right\rangle_{\mathbb{Q}}=\langle 1, e\rangle_{\mathbb{Q}[z]} .
$$

Step 4. By virtue of Theorem 2.7 and Theorem 6.1, we get

$$
h=\frac{\eta^{24}(\tau)}{\eta^{24}(11 \tau) \eta_{11,1}^{28}(\tau) \eta_{11,2}^{16}(\tau) \eta_{11,3}^{12}(\tau) \eta_{11,4}^{4}(\tau)}
$$

for which $h F$ has a pole only at infinity.

Step 5. Employing Radu's Algorithm MC and Algorithm MW, we deduce that $h F \in\left\langle G E^{\infty}(11)\right\rangle_{\mathbb{Q}}$ and

$$
\begin{aligned}
h F=11 z^{10}+121 z^{8} e+330 z^{9}-484 z^{7} e-990 z^{8}+484 z^{6} e+792 z^{7} \\
-484 z^{5} e+44 z^{6}+1089 z^{4} e-132 z^{5}-1452 z^{3} e-451 z^{4} \\
+968 z^{2} e+748 z^{3}-242 z e-429 z^{2}+77 z+11 .
\end{aligned}
$$

This completes the proof.

\section{Further Examples}

In this section, we derive Ramanujan-type identities on the broken 2-diamond partition function. The notion of the broken $k$-diamond partitions was introduced by Andrews and Paule [4] in their study of MacMahon's partition analysis. The number of broken $k$-diamond partitions of $n$ is denoted by $\Delta_{k}(n)$. They showed that the generating function of $\Delta_{k}(n)$ is given by

$$
\sum_{n=0}^{\infty} \Delta_{k}(n) q^{n}=\frac{\left(q^{2} ; q^{2}\right)_{\infty}\left(q^{2 k+1} ; q^{2 k+1}\right)_{\infty}}{(q ; q)_{\infty}^{3}\left(q^{4 k+2} ; q^{4 k+2}\right)_{\infty}}
$$

Andrews and Paule conjectured that

$$
\Delta_{2}(25 n+14) \equiv 0 \quad(\bmod 5) .
$$


Chan [9] proved this conjecture and also showed that

$$
\Delta_{2}(25 n+24) \equiv 0 \quad(\bmod 5)
$$

Define $a(n)$ by

$$
\sum_{n=0}^{\infty} a(n) q^{n}=\frac{(q ; q)_{\infty}^{2}\left(q^{2} ; q^{2}\right)_{\infty}}{\left(q^{10} ; q^{10}\right)_{\infty}}
$$

Since $\left(1-q^{n}\right)^{5} \equiv 1-q^{5 n}(\bmod 5)$, we see that $\Delta_{2}(n) \equiv a(n)(\bmod 5)$. By the Ramanujan-Kolberg algorithm, Radu [35] obtained the following identity:

$$
\begin{array}{r}
\frac{\left(q^{2} ; q^{2}\right)_{\infty}^{12}\left(q^{5} ; q^{5}\right)_{\infty}^{10}}{q^{4}(q ; q)_{\infty}^{6}\left(q^{10} ; q^{10}\right)_{\infty}^{20}}\left(\sum_{n=0}^{\infty} a(25 n+14) q^{n}\right)\left(\sum_{n=0}^{\infty} a(25 n+24) q^{n}\right) \\
=25\left(2 t^{4}+28 t^{3}+155 t^{2}+400 t+400\right)
\end{array}
$$

where

$$
t=\frac{(q ; q)_{\infty}^{3}\left(q^{5} ; q^{5}\right)_{\infty}}{q\left(q^{2} ; q^{2}\right)_{\infty}\left(q^{10} ; q^{10}\right)_{\infty}^{3}}
$$

The congruences (9.1) and (9.2) are easy consequences of (9.3). Let

$$
z=\frac{\left(q^{2} ; q^{2}\right)_{\infty}\left(q^{5} ; q^{5}\right)_{\infty}^{5}}{q(q ; q)_{\infty}\left(q^{10} ; q^{10}\right)_{\infty}^{5}}
$$

Using the package RaduRK, Smoot [46] deduced that

$$
\frac{(q ; q)_{\infty}^{126}\left(q^{5} ; q^{5}\right)_{\infty}^{70}}{q^{58}\left(q^{2} ; q^{2}\right)_{\infty}^{2}\left(q^{10} ; q^{10}\right)_{\infty}^{190}}\left(\sum_{n=0}^{\infty} \Delta_{2}(25 n+14) q^{n}\right)\left(\sum_{n=0}^{\infty} \Delta_{2}(25 n+24) q^{n}\right)
$$

is a polynomial in $z$ of degree 58 with integer coefficients divisible by 25 . It is not hard to see that the above relation implies the congruences (9.1) and (9.2).

Our algorithm provides the following witness identities for $\Delta_{2}(25 n+14)$ and $\Delta_{2}(25 n+24)$.

Theorem 9.1. Let

$$
z=\frac{(q ; q)_{\infty}\left(q^{5} ; q^{5}\right)_{\infty}}{q\left(q, q^{4} ; q^{5}\right)_{\infty}^{2}\left(q^{10} ; q^{10}\right)_{\infty}^{2}\left(q, q^{9} ; q^{10}\right)_{\infty}}
$$

Then

$$
\frac{(q ; q)_{\infty}^{92}\left(q^{5} ; q^{5}\right)_{\infty}^{14}\left(q, q^{4} ; q^{5}\right)_{\infty}^{52}\left(q^{4}, q^{6} ; q^{10}\right)_{\infty}^{4}}{q^{57}\left(q^{2} ; q^{2}\right)_{\infty}^{58}\left(q^{10} ; q^{10}\right)_{\infty}^{46}\left(q, q^{9} ; q^{10}\right)_{\infty}^{109}\left(q^{5} ; q^{10}\right)_{\infty}^{10}} \sum_{n=0}^{\infty} \Delta_{2}(25 n+14) q^{n}
$$

and

$$
\frac{(q ; q)_{\infty}^{92}\left(q, q^{4} ; q^{5}\right)_{\infty}^{62}\left(q^{5} ; q^{10}\right)_{\infty}^{6}}{q^{57}\left(q^{2} ; q^{2}\right)_{\infty}^{59}\left(q^{5} ; q^{5}\right)_{\infty}^{2}\left(q^{10} ; q^{10}\right)_{\infty}^{29}\left(q, q^{9} ; q^{10}\right)_{\infty}^{119}\left(q^{4}, q^{6} ; q^{10}\right)_{\infty}^{4}} \sum_{n=0}^{\infty} \Delta_{2}(25 n+24) q^{n}
$$

are both polynomials in $z$ of degree 57 with integer coefficients divisible by 5 . 
More precisely, (9.4) equals

$$
\begin{aligned}
& 10445 z^{57}+65072505 z^{56}+29885191700 z^{55}+2909565072375 z^{54} \\
& +58232762317950 z^{53}-771909964270635 z^{52}-8976196273201590 z^{51} \\
& +168096305999838525 z^{50}-552704071429548750 z^{49} \\
& -6285133254753356625 z^{48}+76077164750182724400 z^{47} \\
& -350853605818104040400 z^{46}+430844106211910184000 z^{45} \\
& +4332665789140456020000 z^{44}-31965516977695010144000 z^{43} \\
& +116598487085627561478400 z^{42}-254498980254624708134400 z^{41} \\
& + \\
& +226239786150985106784000 z^{40}+630144010340120712320000 z^{39} \\
& -3270835930300215379968000 z^{38}+7873377561448743273881600 z^{37} \\
& -12188753588700934348185600 z^{36}+11409105186984502777856000 z^{35} \\
& -1853370295840331059200000 z^{34}-12922596637778941349888000 z^{33} \\
& +19993842975085327602810880 z^{32}-4136695001339260651438080 z^{31} \\
& - \\
& -
\end{aligned}
$$




$$
\begin{aligned}
& -31960428074332323840 z^{2}-1612499772831170560 z \\
& -7036874417766400 .
\end{aligned}
$$

The explicit expression for (9.5) is omitted.

We end this section by noting that our algorithmic approach can be used to derive dissection formulas on quotients in the form of (1.1), that is,

$$
\prod_{\delta \mid M}\left(q^{\delta} ; q^{\delta}\right)_{\infty}^{r_{\delta}}
$$

where $M$ is a positive integer and $r_{\delta}, r_{\delta, g}$ are integers. Let $a(n)$ be the partition function defined by (1.1), and let $m$ be a positive integer. If our algorithm can be utilized to find a formula for the generating function of $a(m n+t)$ for each $0 \leq t \leq m-1$, then we are led to an $m$-dissection formula on the quotient (9.6). For example, the algorithm is valid to produce the 5-dissection formulas for $(q ; q)_{\infty}$ and $\frac{1}{(q ; q)_{\infty}}$, see Berndt [6, p. 165].

\section{More General Partition Functions}

While many partition functions $a(n)$ are of the form (1.1), there are partition functions that do not seem to fall into this framework, such as Andrews' $(k, i)$-singular overpartition function $\bar{Q}_{k, i}(n)$. Andrews [3] derived the generating function

$$
\sum_{n=0}^{\infty} \bar{Q}_{k, i}(n) q^{n}=\frac{\left(q^{k},-q^{i},-q^{k-i} ; q^{k}\right)_{\infty}}{(q ; q)_{\infty}}
$$

In general, it is not always the case that a quotient on the right hand side of (10.1) can be expressed in the form of (1.1).

The objective of this section is to extend our algorithm to partition functions $b(n)$ defined by

$$
\sum_{n=0}^{\infty} b(n) q^{n}=\prod_{\delta \mid M}\left(q^{\delta} ; q^{\delta}\right)_{\infty}^{r_{\delta}} \prod_{\substack{\delta \mid M \\ 0<g<\delta}}\left(q^{g}, q^{\delta-g} ; q^{\delta}\right)_{\infty}^{r_{\delta, g}}
$$

where $M$ is a positive integer and $r_{\delta}, r_{\delta, g}$ are integers. In fact, for any $k$ and $1 \leq i<\frac{k}{2},(10.1)$ can be written in the form of (10.2):

$$
\sum_{n=0}^{\infty} \bar{Q}_{k, i}(n) q^{n}=\frac{\left(q^{k} ; q^{k}\right)_{\infty}\left(q^{2 i}, q^{2 k-2 i} ; q^{2 k}\right)_{\infty}}{(q ; q)_{\infty}\left(q^{i}, q^{k-i} ; q^{k}\right)_{\infty}}
$$

where $M=2 k$,

$$
r_{\delta}=\left\{\begin{array}{ll}
-1, & \delta=1, \\
1, & \delta=k, \\
0, & \text { otherwise },
\end{array} \quad \text { and } \quad r_{\delta, g}= \begin{cases}-1, & \delta=k, g=i, \\
1, & \delta=2 k, g=2 i \\
0, & \text { otherwise }\end{cases}\right.
$$


Analogous to the generating function $g_{m, t}(\tau)$ in Sect. 2. as given by Radu [37], we adopt the same notation for the generating function of $b(m n+t)$ :

$$
g_{m, t}(\tau)=q^{\frac{t-\ell}{m}} \sum_{n=0}^{\infty} b(m n+t) q^{n},
$$

where

$$
\ell=-\frac{1}{24} \sum_{\delta \mid M} \delta r_{\delta}-\sum_{\substack{\delta \mid M \\ 0<g<\delta}} \frac{\delta}{2} P_{2}\left(\frac{g}{\delta}\right) r_{\delta, g}
$$

As before,

$$
P_{2}(t)=\{t\}^{2}-\{t\}+\frac{1}{6},
$$

and $\{t\}$ is the fractional part of $t$.

To derive a Ramanujan-type identity for $b(m n+t)$, we follow the same procedure as in Sect. 3 .. There are only a few modifications that should be taken into account in order to extend Theorem 2.1 and Theorem 2.7 to the generating function $g_{m, t}(\tau)$ in (10.4). The proofs are similar to those of Theorem 2.1 and Theorem 2.7 and hence are omitted.

Let $\phi(\tau)$ be a generalized eta-quotient and $F=\phi(\tau) g_{m, t}(\tau)$. Similar to Theorems 2.1, we give a criterion for $F(\tau)$ to be a modular function for $\Gamma_{1}(N)$. Let $\kappa=\operatorname{gcd}\left(m^{2}-1,24\right)$. First, we assume that $N$ satisfies the following conditions:

1. $M \mid N$.

2. $p \mid N$ for any prime $p \mid m$.

3. $\kappa N \sum_{\substack{\delta \mid M \\ 0<g<\delta}} \frac{g}{\delta} r_{\delta, g} \equiv 0(\bmod 2)$.

4. $\kappa N \sum_{\substack{\delta \mid M \\ 0<g<\delta}} r_{\delta, g} \equiv 0(\bmod 4)$.

5. $\kappa m N^{2} \sum_{\substack{\delta \mid M \\ 0<g<\delta}} \frac{r_{\delta, g}}{\delta} \equiv 0(\bmod 12)$.

6. $\kappa N \sum_{\delta \mid M} r_{\delta} \equiv 0(\bmod 8)$.

7. $\kappa m N^{2} \sum_{\delta \mid M} \frac{r_{\delta}}{\delta} \equiv 0(\bmod 24)$.

8. $\frac{24 m M}{\operatorname{gcd}(\kappa \alpha(t), 24 m M)} \mid N$, where

$$
\alpha(t)=-M \sum_{\delta \mid M} \delta r_{\delta}-12 M \sum_{\substack{\delta \mid M \\ 0<g<\delta}} \delta P_{2}\left(\frac{g}{\delta}\right) r_{\delta, g}-24 M t .
$$

9. Let $\prod_{\delta \mid M} \delta^{\left|r_{\delta}\right|}=2^{z} j$, where $z \in \mathbb{N}$ and $j$ is odd. If $2 \mid m$, then $\kappa N \equiv 0(\bmod 4)$ and $N z \equiv 0$ $(\bmod 8)$, or $z \equiv 0(\bmod 2)$ and $N(j-1) \equiv 0(\bmod 8)$. 
10. Let $\mathbb{S}_{n}=\left\{j^{2}(\bmod n): j \in \mathbb{Z}_{n}, \operatorname{gcd}(j, n)=1, j \equiv 1(\bmod N)\right\}$. For any $s \in \mathbb{S}_{24 m M}$,

$$
\frac{s-1}{24} \sum_{\delta \mid M} \delta r_{\delta}+(s-1) \sum_{\substack{\delta \mid M \\ 0<g<\delta}} \frac{\delta}{2} P_{2}\left(\frac{g}{\delta}\right) r_{\delta, g}+t s \equiv t \quad(\bmod m) .
$$

For a given partition function $b(n)$, and given integers $m$ and $t$, such a positive integer $N$ always exists, because $N=24 m M$ satisfies the conditions 1-10. For example, for Andrews' (3,1)-singular overpartition function $\bar{Q}_{3,1}(n)$, and for $m=9$ and $t=3$ we have $N=6$. Compared with the conditions in Sect. 2., the conditions $3-5$ are required to deal with the generalized eta-quotients.

Theorem 10.1. For a given partition function $b(n)$ as defined by (10.2), and for given integers $m$ and $t$, suppose that $N$ is a positive integer satisfying the conditions 1-10. Let

$$
F(\tau)=\phi(\tau) g_{m, t}(\tau)
$$

where

$$
\phi(\tau)=\prod_{\delta \mid N} \eta^{a_{\delta}}(\delta \tau) \prod_{\substack{\delta \mid N \\ 0<g \leq\lfloor\delta / 2\rfloor}} \eta_{\delta, g}^{a_{\delta, g}}(\tau)
$$

and $a_{\delta}$ and $a_{\delta, g}$ are integers. Then $F(\tau)$ is a modular function with respect to $\Gamma_{1}(N)$ if and only if $a_{\delta}$ and $a_{\delta, g}$ satisfy the following conditions:

(1) $\sum_{\delta \mid N} a_{\delta}+\sum_{\delta \mid M} r_{\delta}=0$

(2) $N \sum_{\delta \mid N} \frac{a_{\delta}}{\delta}+2 N \sum_{\substack{\delta \mid N \\ 0<g \leq\lfloor\delta / 2\rfloor}} \frac{a_{\delta, g}}{\delta}+N m \sum_{\delta \mid M} \frac{r_{\delta}}{\delta}+2 N m \sum_{\substack{\delta \mid M \\ 0<g<\delta}} \frac{r_{\delta, g}}{\delta} \equiv 0(\bmod 24)$,

(3) $\sum_{\delta \mid N} \delta a_{\delta}+12 \sum_{\substack{\delta \mid N \\ 0<g \leq\lfloor\delta / 2\rfloor}} \delta P_{2}\left(\frac{g}{\delta}\right) a_{\delta, g}+m \sum_{\delta \mid M} \delta r_{\delta}$

$$
+12 m \sum_{\substack{\delta \backslash M \\ 0<g<\delta}} \delta P_{2}\left(\frac{g}{\delta}\right) r_{\delta, g}+\frac{\left(m^{2}-1\right) \alpha(t)}{m M} \equiv 0(\bmod 24),
$$

where

$$
\alpha(t)=-M \sum_{\delta \mid M} \delta r_{\delta}-12 M \sum_{\substack{\delta \mid M \\ 0<g<\delta}} \delta P_{2}\left(\frac{g}{\delta}\right) r_{\delta, g}-24 M t
$$

(4) For any integer $0<a<12 N$ with $\operatorname{gcd}(a, 6)=1$ and $a \equiv 1(\bmod N)$,

$$
\prod_{\delta \mid N}\left(\frac{\delta}{a}\right)^{\left|a_{\delta}\right|} \prod_{\delta \mid M}\left(\frac{m \delta}{a}\right)^{\left|r_{\delta}\right|} e^{\sum_{\delta \mid N} \sum_{g=1}^{\lfloor\delta / 2\rfloor} \pi i\left(\frac{g}{\delta}-\frac{1}{2}\right)(a-1) a_{\delta, g}+\sum_{\delta \mid M} \sum_{g=1}^{\delta-1} \pi i\left(\frac{g}{\delta}-\frac{1}{2}\right)(a-1) r_{\delta, g}}=1 .
$$

In the notation $p(\gamma, \lambda)$ and $p(\gamma)$ in (2.19) and (2.20), we define the map $p: \Gamma \times \mathbb{Z}_{m} \rightarrow \mathbb{Q}$ by

$$
p(\gamma, \lambda)=\frac{1}{24} \sum_{\delta \mid M} \frac{\operatorname{gcd}^{2}(\delta(a+\kappa \lambda c), m c)}{\delta m} r_{\delta}
$$




$$
+\frac{1}{2} \sum_{\substack{\delta \mid M \\ 0<g<\delta}} \frac{\operatorname{gcd}^{2}(\delta(a+\kappa \lambda c), m c)}{\delta m} P_{2}\left(\frac{(a+\kappa \lambda c) g}{\operatorname{gcd}(\delta(a+\kappa \lambda c), m c)}\right) r_{\delta, g},
$$

and define $p(\gamma)$ by

$$
p(\gamma)=\min \{p(\gamma, \lambda): \lambda=0,1, \ldots, m-1\}
$$

Parallel to Theorem 2.7, we obtain lower bounds of the orders of $F(\tau)$ at cusps of $\Gamma_{1}(N)$.

Theorem 10.2. For a given partition function $b(n)$ as defined by (10.2), and for given integers $m$ and $t$, let

$$
F(\tau)=\phi(\tau) g_{m, t}(\tau)
$$

where

$$
\phi(\tau)=\prod_{\delta \mid N} \eta^{a_{\delta}}(\delta \tau) \prod_{\substack{\delta \mid N \\ 0<g \leq\lfloor\delta / 2\rfloor}} \eta_{\delta, g}^{a_{\delta, g}}(\tau),
$$

$a_{\delta}$ and $a_{\delta, g}$ are integers. Assume that $F(\tau)$ is a modular function for $\Gamma_{1}(N)$. Let $\left\{s_{1}, s_{2}, \ldots, s_{\epsilon}\right\}$ be a complete set of inequivalent cusps of $\Gamma_{1}(N)$, and for each $1 \leq i \leq \epsilon$, let $\alpha_{i} \in \Gamma$ be such that $\alpha_{i} \infty=s_{i}$. Then

$$
\operatorname{ord}_{s_{i}}(F(\tau)) \geq w_{\alpha_{i}}\left(p\left(\alpha_{i}\right)+p^{*}\left(\alpha_{i}\right)\right),
$$

where $p(\gamma)$ is given by $(10.5)$ and $p^{*}(\gamma)$ is defined in Lemma 2.6.

For a given partition function $b(n)$, and given integers $m$ and $t$, assume that we have found a generalized eta-quotient $\phi(\tau)$ such that

$$
F(\tau)=\phi(\tau) g_{m, t}(\tau)
$$

is a modular function for $\Gamma_{1}(N)$. Utilizing the algorithm in Sect. 3., we try to express $F(\tau)$ as a linear combination of generalized eta-quotients with level $N$. If we succeed, then we obtain a Ramanujantype identity for $b(m n+t)$. Note that Theorem 10.2 is needed to find a generalized eta-quotient $h(\tau)$ such that $h F$ has a pole only at infinity.

For example, we can derive Ramanujan-type identities on the singular overpartition function introduced by Andrews [3]. The number of $(k, i)$-singular overpartitions of $n$ is denoted by $\bar{Q}_{k, i}(n)$ $\left(1 \leq i<\frac{k}{2}\right)$. For $k=3$ and $i=1,(10.3)$ specializes to

$$
\sum_{n=0}^{\infty} \bar{Q}_{3,1}(n) q^{n}=\frac{\left(q^{3} ; q^{3}\right)_{\infty}\left(q^{2}, q^{4} ; q^{6}\right)_{\infty}}{(q ; q)_{\infty}\left(q, q^{2} ; q^{3}\right)_{\infty}} .
$$

When applied to the above generating function, our algorithm produces the Ramanujan-type identities on $\bar{Q}_{3,1}(9 n+3)$ and $\bar{Q}_{3,1}(9 n+6)$ due to Shen [45].

Theorem 10.3. We have

$$
\frac{(q ; q)_{\infty}^{14}}{q\left(q^{2} ; q^{2}\right)_{\infty}^{5}\left(q^{3} ; q^{3}\right)_{\infty}^{6}\left(q^{6} ; q^{6}\right)_{\infty}^{3}} \sum_{n=0}^{\infty} \bar{Q}_{3,1}(9 n+3) q^{n}=6 z+96,
$$


and

$$
\frac{(q ; q)_{\infty}^{13}}{q\left(q^{2} ; q^{2}\right)_{\infty}^{4}\left(q^{3} ; q^{3}\right)_{\infty}^{3}\left(q^{6} ; q^{6}\right)_{\infty}^{6}} \sum_{n=0}^{\infty} \bar{Q}_{3,1}(9 n+6) q^{n}=24 z+96
$$

where

$$
z=\frac{\left(q^{2} ; q^{2}\right)_{\infty}^{3}\left(q^{3} ; q^{3}\right)_{\infty}^{9}}{q(q ; q)_{\infty}^{3}\left(q^{6} ; q^{6}\right)_{\infty}^{9}}
$$

Our extended algorithm can also be used to derive dissection formulas on the quotients in the form of (10.2), that is,

$$
\prod_{\delta \mid M}\left(q^{\delta} ; q^{\delta}\right)_{\infty}^{r_{\delta}} \prod_{\substack{\delta \mid M \\ 0<g<\delta}}\left(q^{g}, q^{\delta-g} ; q^{\delta}\right)_{\infty}^{r_{\delta, g}}
$$

where $M$ is a positive integer and $r_{\delta}, r_{\delta, g}$ are integers. Let $b(n)$ be the partition function defined by (10.2), and let $m$ be a positive integer. If our algorithm can be utilized to find a formula for the generating function of $b(m n+t)$ for each $0 \leq t \leq m-1$, then we are led to an $m$-dissection formula on the quotient in (10.8). For example, we get the 2-, 4-dissections of the Rogers-Ramanujan continued fraction $[2,21,27,40]$, the 8-dissections of the Gordon's continued fraction [22,49] and the 2-, 3-, 4-, 6 -dissections of Ramanujan's cubic continued fraction [23, 47].

We now demonstrate how to deduce the 2-dissection formula for the Rogers-Ramanujan continued fraction:

$$
R(q)=\frac{1}{1}+\frac{q}{1}+\frac{q^{2}}{1}+\frac{q^{3}}{1}+\cdots .
$$

Rogers [42, p. 329] showed that

$$
R(q)=\frac{\left(q^{2}, q^{3} ; q^{5}\right)_{\infty}}{\left(q, q^{4} ; q^{5}\right)_{\infty}}
$$

The following 2-dissection formulas of Ramanujan [40, p. 50] were first proved by Andrews [2]. With respect to the quotient in (10.9), we have to count on the extended algorithm because (10.9) cannot be expressed in the form of (1.1).

Theorem 10.4. We have

$$
R(q)=\frac{\left(q^{8}, q^{12} ; q^{20}\right)_{\infty}^{2}}{\left(q^{6}, q^{14} ; q^{20}\right)_{\infty}\left(q^{10}, q^{10} ; q^{20}\right)_{\infty}}+q \frac{\left(q^{2}, q^{18} ; q^{20}\right)_{\infty}\left(q^{8}, q^{12} ; q^{20}\right)_{\infty}}{\left(q^{4}, q^{16} ; q^{20}\right)_{\infty}\left(q^{10}, q^{10} ; q^{20}\right)_{\infty}}
$$

and

$$
R(q)^{-1}=\frac{\left(q^{4}, q^{16} ; q^{20}\right)_{\infty}^{2}}{\left(q^{2}, q^{18} ; q^{20}\right)_{\infty}\left(q^{10}, q^{10} ; q^{20}\right)_{\infty}}-q \frac{\left(q^{4}, q^{16} ; q^{20}\right)_{\infty}\left(q^{6}, q^{14} ; q^{20}\right)_{\infty}}{\left(q^{8}, q^{12} ; q^{20}\right)_{\infty}\left(q^{10}, q^{10} ; q^{20}\right)_{\infty}}
$$

Proof. As far as (10.9) is concerned, we have $M=5, r_{5,1}=-1$ and $r_{5,2}=1$. We find that $N=10$ satisfies the conditions $1-10$. Let $r(n)$ be defined by

$$
R(q)=\sum_{n=0}^{\infty} r(n) q^{n}
$$


Employing our algorithm, we obtain that

$$
\sum_{n=0}^{\infty} r(2 n) q^{n}=\frac{z_{1} z_{3}}{z_{2} z^{2}} \cdot \frac{\eta_{10,5}^{2}(\tau)}{\eta_{10,4}^{2}(\tau)}
$$

and

$$
\sum_{n=0}^{\infty} r(2 n+1) q^{n}=\frac{z_{2}^{3} z^{4}}{z_{1}^{2} z_{3}^{3}} \cdot \frac{\eta_{10,4}^{8}(\tau)}{\eta_{10,5}^{8}(\tau)}
$$

where $z, z_{1}, z_{2}$ and $z_{3}$ are given in (3.2). A direct computation yields (10.10). Similarly, we get (10.11). This completes the proof.

Gordon [17] showed that

$$
1+q+\frac{q^{2}}{1+q^{3}}+\frac{q^{4}}{1+q^{5}}+\frac{q^{6}}{1+q^{7}}+\ldots=\frac{\left(q^{3}, q^{5} ; q^{8}\right)_{\infty}}{\left(q, q^{7} ; q^{8}\right)_{\infty}} .
$$

Using our algorithm, we deduce the following 8-dissection formulas of Hirschhorn for (10.12) and its reciprocal, see [22, pp. 373-374].

Theorem 10.5 (Hirschhorn [22]). We have

$$
\begin{aligned}
\frac{\left(q^{3}, q^{5} ; q^{8}\right)_{\infty}}{\left(q, q^{7} ; q^{8}\right)_{\infty}}=\frac{\left(-q^{24},-q^{32},-q^{32},-q^{40}, q^{64}, q^{64} ; q^{64}\right)_{\infty}}{\left(q^{8}, q^{16}, q^{16}, q^{24}, q^{32}, q^{32} ; q^{32}\right)_{\infty}} & +q \frac{\left(-q^{16},-q^{24},-q^{40},-q^{48}, q^{64}, q^{64} ; q^{64}\right)_{\infty}}{\left(q^{16}, q^{8}, q^{24}, q^{24}, q^{48}, q^{64}, q^{64} ; q^{64}\right)_{\infty}} \\
& +q^{2} \frac{\left(-q^{16},-q^{24},-q^{40},-q^{48}, q^{64}, q^{64} ; q^{64}\right)_{\infty}}{\left(q^{8}, q^{16}, q^{16}, q^{24}, q^{32}, q^{64} ; q^{64} ; q^{64}\right)_{\infty}} \\
& -2 q^{12} \frac{\left(-q^{8},-q^{16},-q^{64},-q^{64}, q^{64}, q^{64} ; q^{64}\right)_{\infty}}{\left(q^{8}, q^{16}, q^{16}, q^{24}, q^{32}, q^{32} ; q^{32}\right)_{\infty}} \\
& -q^{5} \frac{\left(-q^{8},-q^{16},-q^{48},-q^{56}, q^{64}, q^{64} ; q^{64}\right)_{\infty}}{\left(q^{8}, q^{8}, q^{24}, q^{24}, q^{32}, q^{32} ; q^{32}\right)_{\infty}} \\
& -q^{6} \frac{\left(-q^{8},-q^{16},-q^{48},-q^{56}, q^{64}, q^{64} ; q^{64}\right)_{\infty}}{\left(q^{8}, q^{16}, q^{16}, q^{24}, q^{32}, q^{32} ; q^{32}\right)_{\infty}} \\
\frac{\left(q, q^{7} ; q^{8}\right)_{\infty}}{\left(q^{3}, q^{5} ; q^{8}\right)_{\infty}}= & \left.-q^{16},-q^{24},-q^{40},-q^{48}, q^{64}, q^{64} ; q^{64}\right)_{\infty} \\
& \left(q^{8}, q^{8}, q^{24}, q^{24}, q^{32}, q^{32} ; q^{32}\right)_{\infty} \\
& -q \frac{\left(-q^{16},-q^{24},-q^{40},-q^{48}, q^{64}, q^{64} ; q^{64}\right)_{\infty}}{\left(q^{8}, q^{16}, q^{16}, q^{24}, q^{32}, q^{32} ; q^{32}\right)_{\infty}} \\
& +q^{3} \frac{\left(-q^{8},-q^{32},-q^{32},-q^{56}, q^{64}, q^{64} ; q^{64}\right)_{\infty}}{\left(q^{8}, q^{16}, q^{16}, q^{24}, q^{32}, q^{32} ; q^{32}\right)_{\infty}}
\end{aligned}
$$




$$
\begin{aligned}
& -q^{4} \frac{\left(-q^{8},-q^{16},-q^{48},-q^{56}, q^{64}, q^{64} ; q^{64}\right)_{\infty}}{\left(q^{8}, q^{8}, q^{24}, q^{24}, q^{32}, q^{32} ; q^{32}\right)_{\infty}} \\
& +q^{5} \frac{\left(-q^{8},-q^{16},-q^{48},-q^{56}, q^{64}, q^{64} ; q^{64}\right)_{\infty}}{\left(q^{8}, q^{16}, q^{16}, q^{24}, q^{32}, q^{32} ; q^{32}\right)_{\infty}} \\
& -2 q^{7} \frac{\left(-q^{24},-q^{40},-q^{64},-q^{64}, q^{64}, q^{64} ; q^{64}\right)_{\infty}}{\left(q^{8}, q^{16}, q^{16}, q^{24}, q^{32}, q^{32} ; q^{32}\right)_{\infty}} .
\end{aligned}
$$

Ramanujan's cubic continued fraction is defined by

$$
\frac{1}{1}+\frac{q+q^{2}}{1}+\frac{q^{2}+q^{4}}{1}+\cdots
$$

which equals

$$
\frac{\left(q, q^{5} ; q^{6}\right)_{\infty}}{\left(q^{3}, q^{3} ; q^{6}\right)_{\infty}}
$$

see [40, p. 44]. Applying our algorithm to (10.13) and its reciprocal, we are led to the 2-, 3-, 4- and 6-dissection formulas in Theorem 1.1-Theorem 1.4 in [23].

\section{Acknowledgements}

We are grateful to Peter Paule for his inspiring lectures and for stimulating discussions. We would also like to thank the referees for their valuable comments and suggestions.

\section{References}

[1] 4ti2 team: 4ti2 - A software package for algebraic, geometric and combinatorial problems on linear spaces software. Available at https://4ti2.github.io

[2] Andrews, G.E.: Ramunujan's "lost" notebook III. The Rogers-Ramanujan continued fraction. Adv. Math. 41, 186-208 (1981)

[3] Andrews, G.E.: Singular overpartitions. Int. J. Number Theory 11(5), 1523-1533 (2015)

[4] Andrews, G.E., Paule, P.: MacMahon's partition analysis XI: Broken diamonds and modular forms. Acta Arith. 126(3), 281-294 (2007)

[5] Atkin, A.O.L., Swinnerton-Dyer, P.: Some properties of partitions. Proc. London Math. Soc. (3) 4, 84-106 (1954)

[6] Berndt, B.C.: Number Theory in the Spirit of Ramanujan. Student Mathematical Library, 34. Amer. Math. Soc., Providence, RI (2006)

[7] Bilgici, G., Ekin, A.B.: Some congruences for modulus 13 related to partition generating function. Ramanujan J. 33(2), 197-218 (2014) 
[8] Bilgici, G., Ekin, A.B.: 11-Dissection and modulo 11 congruences properties for partition generating function. Int. J. Contemp. Math. Sci. 9(1-4), 1-10 (2014)

[9] Chan, S.H.: Some congruences for Andrews-Paule's broken 2-diamond partitions. Discrete Math. $308(23), 5735-5741$ (2008)

[10] Cho, B., Koo, J.K., Park, Y.K.: Arithmetic of the Ramanujan-Göllnitz-Gordon continued fraction. J. Number Theory 129(4), 922-947 (2009)

[11] Corteel, S., Lovejoy, J.: Overpartitions. Trans. Amer. Math. Soc. 356(4), 1623-1635 (2004)

[12] Diamond, F., Shurman, J.: A First Course in Modular Forms. Graduate Texts in Mathematics, 228. Springer-Verlag, New York (2005)

[13] Eichhorn, D.A.: Some results on the congruential and gap-theoretic study of partition functions. Ph.D. Thesis. University of Illinois at Urbana-Champaign (1999)

[14] Eichhorn, D.A., Ono, K.: Congruences for partition functions. In: Berndt, B.C., Diamond, H.G., Hildebrand, A.J. (eds.) Analytic Number Theory, Vol. 1 (Allerton Park, IL, 1995), pp. 309-321. Progr. Math., 138, Birkhäuser Boston, Boston, MA (1996)

[15] Eichhorn, D.A., Sellers, J.A.: Computational proofs of congruences for 2-colored Frobenius partitions. Int. J. Math. Math. Sci. 29(6), 333-340 (2002)

[16] Gasper, G., Rahman, M.: Basic Hypergeometric Series. Second Edition. Encyclopedia of Mathematics and its Applications, 96. Cambridge University Press, Cambridge (2004)

[17] Gordon, B.: Some continued fractions of the Rogers-Ramanujan type. Duke Math. J. 32, 741-748 (1965)

[18] Hardy, G.H.: Note on Ramanujan's arithmetic function $\tau(n)$. Proc. Cambridge Philos. Soc. 23, 675-680 (1927)

[19] Hardy, G.H.: A further note on Ramanujan's arithmetic function $\tau(n)$. Proc. Cambridge Philos. Soc. 34, 309-315 (1938)

[20] Hemmecke, R.: Dancing samba with Ramanujan partition congruences. J. Symbolic Comput. 84, $14-24(2018)$

[21] Hirschhorn, M.D.: On the expansion of Ramanujan's continued fraction. Ramanujan J. 2(4), $521-527(1998)$

[22] Hirschhorn, M.D.: On the expansion of a continued fraction of Gordon. Ramanujan J. 5(4), $369-375$ (2001)

[23] Hirschhorn, M.D., Roselin: On the 2-, 3-, 4- and 6-dissections of Ramanujan's cubic continued fraction and its reciprocal. In: Baruah, N.D., Berndt, B.C., Cooper, S., Huber, T., Schlosser, M.J. (eds.) Ramanujan Rediscovered, pp. 125-138. Ramanujan Math. Soc. Lect. Notes Ser., 14, Ramanujan Math. Soc., Mysore (2010)

[24] Hirschhorn, M.D., Sellers, J.A.: Arithmetic relations for overpartitions. J. Combin. Math. Combin. Comput. 53, 65-73 (2005)

[25] Knopp, M.: Modular Functions in Analytic Number Theory. Second Edition. Amer. Math. Soc., Chelsea Publishing (1993) 
[26] Kolberg, O.: Some identities involving the partition function. Math. Scand. 5, 77-92 (1957)

[27] Lewis, R., Liu, Z.-G.: A conjecture of Hirschhorn on the 4-dissection of Ramanujan's continued fraction. Ramanujan J. 4(4), 347-352 (2000)

[28] Newman, M.: Construction and application of a class of modular functions. Proc. London. Math. Soc. (3) 7, 334-350 (1957)

[29] Newman, M.: Construction and application of a class of modular functions (II). Proc. Lond. Math. Soc. (3) 9, 373-387 (1959)

[30] Paule, P., Radu, C.-S.: A unified algorithmic framework for Ramanujan's congruences modulo powers of 5, 7, and 11. Preprint (2018)

[31] Paule, P., Radu, C.-S.: A new witness identity for $11 \mid p(11 n+6)$. In: Andrews, G.E., Garvan, F. (eds.) Analytic Number Theory, Modular Forms and $q$-Hypergeometric Series, Springer Proc. Math. Stat., 221, pp. 625-639. Springer, Cham (2017)

[32] Paule, P., Radu, S.: Partition analysis, modular functions, and computer algebra. In: Beveridge, A., Griggs, J.R., Hogben, L., Musiker, G., Tetali, P. (eds.) Recent Trends in Combinatorics, IMA Vol. Math. Appl., 159, pp. 511-543. Springer, Cham (2016)

[33] Rademacher, H.: The Ramanujan identities under modular substitutions. Trans. Amer. Math. Soc. 51, 609-636 (1942)

[34] Rademacher, H.: Topics in Analytic Number Theory. Die Grundlehren der mathematischen Wissenschaften, Band 169. Springer-Verlag, New York-Heidelberg (1973)

[35] Radu, C.-S.: An algorithmic approach to Ramanujan-Kolberg identities. J. Symbolic Comput. $68(1), 225-253(2015)$

[36] Radu, S.: An algorithmic approach to Ramanujan's congruences and related problems. Ph.D. Thesis. Research Institute for Symbolic Computation Johannes Kepler University, Linz (2009)

[37] Radu, S.: An algorithmic approach to Ramanujan's congruences. Ramanujan J. 20(2), 215-251 (2009)

[38] Ramanujan, S.: Some properties of $p(n)$, the number of partitions of $n$. Proc. Cambridge Philos. Soc. 19, 207-210 (1919)

[39] Ramanujan, S.: On certain arithmetical functions. Trans. Cambridge Philos. Soc. 22, 159-184 (1916)

[40] Ramanujan, S.: The Lost Notebook and Other Unpublished Papers. Narosa Publishing House, New Delhi (1988)

[41] Robins, S.: Generalized Dedekind $\eta$-products. In: Andrews, G.E., Bressoud, D.M., Parson, L.A. (eds.) The Rademacher Legacy to Mathematics (University Park, PA, 1992), Contemp. Math., 166, pp. 119-128. Amer. Math. Soc., Providence, RI (1994)

[42] Rogers, L.J.: Second memoir on the expansion of certain infinite products. Proc. London Math. Soc. 25, 318-343 (1894)

[43] Schoeneberg, B.: Elliptic Modular Functions: An Introduction. Die Grundlehren der mathematischen Wissenschaften, Band 203. Springer-Verlag, New York-Heidelberg (1974) 
[44] Schrijver, A.: Theory of Linear and Integer Programming. Wiley-Interscience Series in Discrete Mathematics. John Wiley \& Sons, Ltd., Chichester (1986)

[45] Shen, E.Y.Y.: Arithmetic properties of l-regular overpartitions. Int. J. Number Theory 12(3), $841-852(2016)$

[46] Smoot, N.A.: An implementation of Radu's Ramanujan-Kolberg algorithm. RISC Technical Report (2019)

[47] Srivastava, B.: On 2-dissection and 4-dissection of Ramanujan's cubic continued fraction and identities. Tamsui Oxf. J. Math. Sci. 23(3), 305-315 (2007)

[48] Stein, W.: Modular Forms, A Computational Approach. Graduate Studies in Mathematics, 79. Amer. Math. Soc., Providence, RI (2007)

[49] Xia, E.X.W., Yao, X.M.: The 8-dissection of the Ramanujan-Göllnitz-Gordon continued fraction by an iterative method. Int. J. Number Theory 7(6), 1589-1593 (2011)

[50] Zuckerman, H.S.: Identities analogous to Ramanujan's identities involving the partition function. Duke Math. J. 5(1), 88-110 (1939) 